\title{
The Hera Saturn Entry Probe Mission
}

O. Mousis, D.H. Atkinson, T. Spilker, E. Venkatapathy, J. Poncy, R. Frampton, A. Coustenis, K. Reh, J.-P. Lebreton, L. Fletcher, R. Hueso, M. Amato, A. Colaprete, F. Ferri, D. Stam, P. Wurz, S. Atreya, S. Aslam, D. Banfield, S. Calcutt, G. Fischer, A. Holland, C. Keller, E. Kessler, M. Leese, P. Levacher, A. Morse, O. Munoz, J.-B. Renard, S. Sheridan, F.-X. Schmider, F. Snik, J. H. Waite, M. Bird, T. Cavalié, M. Deleuil, J. Fortney, D. Gautier, T. Guillot, J. I. Lunine, B. Marty, C. Nixon, G.

S. Orton, A. Sánchez-Lavega

O. Mousis, M. Deleuil, P. Levacher

Aix Marseille Université, CNRS, LAM (Laboratoire d'Astrophysique de Marseille) UMR 7326, 13388, Marseille, France, e-mail: olivier.mousis@lam.fr

D. H. Atkinson

Department of Electrical and Computer Engineering, University of Idaho, Moscow, Idaho, USA

T. Spilker

Solar System Science \& Exploration, Monrovia, USA

E. Venkatapathy, A. Colaprete

NASA Ames Research Center, Moffett Field, California, USA

J. Poncy

Thales Alenia Space, Cannes, France

R. Frampton

The Boeing Company, Huntington Beach, California, USA

A. Coustenis, D. Gautier

LESIA, Observatoire de Paris, CNRS, UPMC, Univ. Paris-Diderot, France

K. Reh, G. S. Orton

Jet Propulsion Laboratory, California Institute of Technology, 4800 Oak Grove Dr., Pasadena, CA 91109, USA

J.-P. Lebreton

LPC2E, CNRS-Université d'Orléans, 3a Avenue de la Recherche Scientifique, 45071 Orléans Cedex 2, France

LESIA, Observatoire de Paris, CNRS, UPMC, Univ. Paris-Diderot, France

L. N. Fletcher, S. B. Calcutt

Atmospheric, Oceanic and Planetary Physics, Clarendon Laboratory, University of Oxford, Parks Road, Oxford OX1 3PU, UK

R. Hueso, A. Sánchez-Lavega

Departamento Física Aplicada I, Universidad del País Vasco UPV/EHU, ETS Ingeniería, Alameda Urquijo s/n, 48013 Bilbao, Spain

Unidad Asociada Grupo Ciencias Planetarias UPV/EHU-IAA(CSIC), 48013 Bilbao, Spain

M. Amato, S. Aslam, C. A. Nixon

NASA Goddard Space Flight Center, Greenbelt, MD 20771, USA

F. Ferri

Università degli Studi di Padova, Centro di Ateneo di Studi e Attività Spaziali "Giuseppe Colombo" (CISAS), via Venezia 15, 35131 Padova, Italy 
Abstract The Hera Saturn entry probe mission is proposed as an M-class mission led by ESA with a contribution from NASA. It consists of one atmospheric probe to be sent into the atmosphere of Saturn, and a Carrier-Relay spacecraft. In this concept, the Hera probe is designed from ESA and NASA elements, and the Carrier-Relay Spacecraft is delivered by ESA. The probe is powered by batteries,

D. Stam

Aerospace Engineering, Technical University, Delft, the Netherlands

P. Wurz

Space Science \& Planetology, Physics Institute, University of Bern, Sidlerstrasse 5, 3012 Bern, Switzerland

S. Atreya

Department of Atmospheric, Oceanic, and Space Sciences, University of Michigan, Ann Arbor, MI 48109-2143, USA

D. Banfield, J. I. Lunine

Center for Radiophysics and Space Research, Space Sciences Building Cornell University, Ithaca, NY 14853, USA

G. Fischer

Space Research Institute, Austrian Academy of Sciences, Schmiedlstrasse 6, A-8042 Graz, Austria

A. Holland, A. Morse, S. Sheridan, M. Leese

Department of Physical Sciences, The Open University, Walton Hall, Milton Keynes MK7 6AA, UK

C. Keller, F. Snik

Leiden Observatory, Leiden University, The Netherlands

E. Kessler

Max-Planck-Institut für Quantenoptik, Garching, Germany

O. Munoz

Instituto de Astrofísica de Andalucía, Granada, Spain

J.-B. Renard

LPC2E, CNRS-Université d'Orléans, 3a Avenue de la Recherche Scientifique, 45071 Orléans Cedex 2, France

F.-X. Schmider, T. Guillot

Observatoire de la Côte d'Azur, Laboratoire Lagrange, BP 4229, 06304 Nice cedex 4, France

J. H. Waite

Southwest Research Institute, San Antonio, TX 78228, USA

M. Bird

University of Bonn, Bonn, Germany

T. Cavalié

Max-Planck-Institut für Sonnensystemforschung, Justus von Liebig Weg 3, 37077 Göttingen, Germany

J. Fortney

University of California/Santa Cruz, California, USA

B. Marty

CRPG-CNRS, Nancy-Université, 15 rue Notre Dame des Pauvres, 54501 Vandoeuvre-ls-Nancy, France 
and the Carrier-Relay Spacecraft is powered by solar panels and batteries. We anticipate two major subsystems to be supplied by the United States, either by direct procurement by ESA or by contribution from NASA: the solar electric power system (including solar arrays and the power management and distribution system), and the probe entry system (including the thermal protection shield and aeroshell). Hera is designed to perform in situ measurements of the chemical and isotopic compositions as well as the dynamics of Saturn's atmosphere using a single probe, with the goal of improving our understanding of the origin, formation, and evolution of Saturn, the giant planets and their satellite systems, with extrapolation to extrasolar planets. Hera's aim is to probe well into the cloud-forming region of the troposphere, below the region accessible to remote sensing, to the locations where certain cosmogenically abundant species are expected to be well mixed. By leading to an improved understanding of the processes by which giant planets formed, including the composition and properties of the local solar nebula at the time and location of giant planet formation, Hera will extend the legacy of the Galileo and Cassini missions by further addressing the creation, formation, and chemical, dynamical, and thermal evolution of the giant planets, the entire solar system including Earth and the other terrestrial planets, and formation of other planetary systems.

Key words: Saturn - Atmosphere - Probe - in situ measurements - ESA M4 call

\section{Introduction}

The Hera Saturn entry probe mission is proposed as an M-class mission led by ESA with a significant contribution from NASA. It consists of one atmospheric probe to be sent into the atmosphere of Saturn, and a Carrier-Relay spacecraft (hereafter CRSC). Hera will perform in situ measurements of the chemical and isotopic compositions as well as the dynamics of Saturn's atmosphere using a single probe, with the goal of improving our understanding of the origin, formation, and evolution of Saturn, the giant planets and the solar system. Hera will probe well into the cloudforming region of the troposphere, below the region accessible to remote sensing, to the locations where certain cosmogenically abundant species are expected to be well mixed.

The formation and evolution of the giant planets hold many keys to understanding the formation and evolution of the solar system as a whole, including the terrestrial planets, as well as exoplanetary systems. Key measurements include the composition and processes within giant planet atmospheres, gravitational fields, magnetospheres, and systems of moons. The Galileo probe provided in situ measurements of the chemical and isotopic composition of Jupiter's atmosphere. Of particular importance, the Jovian helium abundance was determined with a high accuracy. Moreover, the Galileo probe revealed unexpected enrichments of the noble gases $\mathrm{Ar}, \mathrm{Kr}$ and Xe with respect to the solar abundances. Additionally, the Galileo probe mass spectrometer measured the ${ }^{14} \mathrm{~N} /{ }^{15} \mathrm{~N}$ ratio, which strongly suggested that the nitrogen in 
Jupiter's atmosphere was acquired from the protosolar nebula (hereafter PSN). The Galileo probe and orbiter mission to Jupiter, complemented by the Juno mission currently en route to Jupiter and the L-class JUICE mission selected by ESA, will provide a solid understanding of the Jupiter system. The Cassini orbiter is providing valuable observations of Saturn's upper atmosphere, system of moons, gravitational field, and magnetosphere. However, the Huygens probe did not explore Saturn's atmosphere. The key missing element towards a similar system understanding of Saturn and an improved context for understanding the Galileo, Juno, and JUICE studies of Jupiter are the measurements of the composition of and the processes within Saturn's deeper atmosphere that only in situ exploration can provide. The Hera probe will use mass spectrometry to measure the abundances of hydrogen, helium, neon, argon, krypton, xenon, carbon, nitrogen, sulfur, and their compounds at near-equatorial latitudes down to at least 10 bars. During its descent, Hera will also sample key isotopic ratios $\mathrm{D} / \mathrm{H},{ }^{3} \mathrm{He} / 4 \mathrm{He},{ }^{20} \mathrm{Ne} /{ }^{21} \mathrm{Ne} /{ }^{22} \mathrm{Ne},{ }^{36} \mathrm{Ar} /{ }^{38} \mathrm{Ar},{ }^{12} \mathrm{C} /{ }^{13} \mathrm{C}$, ${ }^{14} \mathrm{~N} /{ }^{15} \mathrm{~N},{ }^{16} \mathrm{O} /{ }^{17} \mathrm{O} /{ }^{18} \mathrm{O},{ }^{82} \mathrm{Kr} /{ }^{83} \mathrm{Kr} /{ }^{84} \mathrm{Kr} /{ }^{86} \mathrm{Kr}$, and ${ }^{129} \mathrm{Xe} /{ }^{130} \mathrm{Xe} /{ }^{132} \mathrm{Xe} /{ }^{134} \mathrm{Xe} /{ }^{136} \mathrm{Xe}$. In situ measurements of Saturn's well-mixed atmosphere gases will provide a vital comparison to the Galileo probe measurements at Jupiter, and a crucial "ground truth" for the remote sensing investigations by the Cassini orbiter. Hera will investigate Saturn's atmospheric dynamics along its descent trajectory, from (1) the vertical distribution of the pressure, temperature, clouds and wind speeds, and (2) deep wind speeds, differential rotation and convection, by combining probe, gravity and radiometric measurements. Hera is the next logical step in our exploration of the Gas Giants beyond the Galileo and Cassini missions.

Hera will lead to an improved understanding of the processes responsible for the formation of giant planets (contribution of the local solar nebula, accretion of icy planetesimals, and nature and formation temperature of the latter). The Hera data will shed light on the composition of giant planet precursors and on the dynamical evolution of the early solar system. Hera will also address the question as to why Jupiter and Saturn are so different in size, density and core dimension, investigating different pathways to planetary formation, thereby providing new insights on the mechanisms that led to the stunning diversity of giant planets.

The Hera probe will be designed from ESA and NASA elements, and the CRSC will be delivered by ESA. The probe will be powered by batteries. The CRSC will be powered by solar panels and batteries. We anticipate two subsystems to be supplied by the United States, either by direct procurement by ESA or by contribution from NASA: the solar electric power system (including solar arrays and the power management and distribution system), and the probe entry system (including the thermal protection shield and aeroshell). Following the highly successful example of the Cassini-Huygens mission, Hera will carry European and American instruments, with scientists and engineers from both agencies and many affiliates participating in all aspects of mission development and implementation. A Saturn probe is one of the five missions on the NASA New Frontier's list, making Hera a high priority for the European and American Planetary Science communities.

The proposed launch window for Hera is in May 2025. Hera flight begins with a Soyuz-Fregat launch from Kourou on a transfer trajectory to Saturn via several 
inner solar system flybys with an arrival at Saturn in August 2033. The Hera CRSC releases the probe on a ballistic trajectory that will take it into Saturn's atmosphere a few weeks later. Prior to probe release, the CRSC will image Saturn to provide a global context for the probe science, as well as providing a local context of the probe entry location. Following the release of the Hera probe, the CRSC is deflected to prepare for flight over the probe entry location for the probe data relay.

The science objectives and instruments requirements of the mission are described in Sec. 2. The proposed science instruments are detailed in Sec. 3. Section 4 is dedicated to a description of the mission configuration and profile. We discuss the management scheme and mission costs in Sec. 5 and 6, respectively. Sec. 7 is devoted to summary and conclusion.

\section{Science Objectives And Requirements}

\subsection{Context}

The giant planets Jupiter, Saturn, Uranus and Neptune contain most of the mass and angular momentum of the sun's planetary system, and have played a significant role in shaping the solar system's large-scale architecture and evolution, as well as the properties of the smaller, inner worlds [22]. In particular, the formation of these planets has affected the timing and efficiency of volatile delivery to the Earth and other terrestrial planets [12]. Understanding giant planet formation is therefore essential for understanding the origin and evolution of the Earth and other potentially habitable bodies within the solar system. The origin of the giant planets, their influence on the architecture of planetary systems, and the plethora of physical and chemical processes within their atmospheres, make the giant planets particularly important destinations for exploration.

Both Jupiter and Saturn, the gas giants, are thought to have relatively small cores surrounded by massive envelopes composed primarily of hydrogen and helium. Uranus and Neptune are called ice giants because their density is consistent with the presence of a significant fraction of ices/rocks in their interiors. Despite the apparent grouping into two classes in the solar system, giant planets likely exist on a continuum, each carrying the characteristics of their particular formation environment. Comparative planetology of the sun's four giants is therefore essential to reveal the formational, migrational, and evolutionary processes during the early ages of the PSN.

The scientific goals of Hera are fully detailed in [28]. The in situ exploration of Saturn's atmosphere addresses two themes that reach far beyond the unique knowledge gained about an individual planet, including (i) the formation history of the solar system and extrasolar planetary systems, and (ii) the processes that affect the vertical structure of temperatures, clouds and gaseous composition in planetary atmospheres. Examples of the latter are the stochastic and positional variances within 
the PSN, the depth of atmospheric zonal winds, the propagation of atmospheric waves, the formation of clouds and hazes, and disequilibrium processes of photochemistry and vertical mixing that are common to all planetary atmospheres, from terrestrial planets to gas and ice giants and from brown dwarfs to exoplanets.

\subsection{Why In Situ Measurements Are Needed?}

We have obtained most of our knowledge on the physical properties of the sun's giant planets through remote sensing from orbiters, fly-by missions, and ground-based telescopes. At visible wavelengths, remote sensing captures scattered and reflected sunlight, with a penetration depth into an atmosphere down to the upper hazes and clouds, At longer wavelengths, the thermal radiation from deeper layers emerges from the top of the planetary atmosphere. Indeed, important physical data addressing planetary composition, structure, and dynamics can be obtained with an orbiting spacecraft, as illustrated by the successful Galileo and Cassini missions. The information content of remote sensing data, however, remains severely limited due to (i) the degeneracies between the effects of temperatures, clouds, hazes, and gas abundances on the emergent spectra, and (ii) the limited penetration depth and vertical resolution.

As an example of the latter, the vertical distribution of many gases is strongly determined by chemical and condensation processes: many of the most common elements are locked away in a condensed phase, such as clouds or hazes in the deeper troposphere, hiding the main volatile reservoir from the reaches of remote sensing. The abundances of these gases in the upper atmospheric regions as derived from remote sensing data will thus not be representative of the bulk reservoir. Examples are $\mathrm{NH}_{3}$ and $\mathrm{H}_{2} \mathrm{~S}$ (that will form $\mathrm{NH}_{4} \mathrm{SH}$ clouds), $\mathrm{H}_{2} \mathrm{O}$, and other minor species such as $\mathrm{PH}_{3}, \mathrm{AsH}_{3}, \mathrm{GeH}_{4}$ and tropospheric $\mathrm{CO}$. Only by penetrating the "visible" weather layer, with the stratospheric hazes and upper clouds, can we sample the deeper troposphere and determine the true atmospheric composition. With in situ measurements, we will also be able to retrieve the vertical distribution of the lower tropospheric clouds and hazes, and the microphysical properties (size, shape, composition) of their particles that not only act as storage for elements, but also strongly influence the radiation field, the chemical and dynamical processes.

With in situ measurements, we can also trace the vertical dynamics that play a role in gas distributions. An example of the latter is the $\mathrm{PH}_{3}$ profile, where the competing processes of photochemical sinks at high altitudes and sources from below could give a variety of profiles, depending on such factors as the strength of vertical upwelling. Also, a descending probe remains the only direct technique for measuring wind speeds at depths below the visible clouds.

Some species such as the heavier noble gases do not leave distinct traces in spectra measured with remote sensing techniques and for these gases, in situ measurements are the only option to retrieve their abundances. A remarkable example of the capability of in situ probe measurements is illustrated by the exploration of Jupiter, 
where key data regarding the noble gas abundances and the helium mixing ratio could only be obtained through measurements by the Galileo probe [32].

The Galileo probe measurements provided new insights into the formation of the solar system. In particular, the Jovian helium abundance was precisely determined with an accuracy of $2 \%$ [44]; an accuracy impossible to achieve with remote sensing. An accurate measurement of the helium abundance in the atmospheres of giant planets is a key step towards understanding the fundamental problem of their formation and evolution in the solar and extrasolar systems. Moreover, the Galileo probe revealed the unexpected enrichments of $\mathrm{Ar}, \mathrm{Kr}$ and Xe with respect to their solar abundances, suggesting different scenarios for Jupiter's formation (see Sec. B.3.3). Another important result provided by the Galileo probe mass spectrometer was the ${ }^{14} \mathrm{~N} /{ }^{15} \mathrm{~N}$ ratio, a value that suggested that Jupiter acquired its $\mathrm{N}_{2}$ from the PSN reservoir.

The Galileo probe was designed to reach a depth of 10 bars, but survived to pressures exceeding 22 bars, descending into a region depleted in volatiles and gases by unusual "hot spot" meteorology $[31,46]$. Therefore, the Galileo probe measurements of $\mathrm{H}_{2} \mathrm{O}$ abundances are unlikely to represent Jupiter's bulk composition. These measurements nevertheless allowed us a giant step forward in understanding Jupiter. However, the solar-system chemical inventory and formation processes cannot be truly understood from the measured elemental and isotopic enrichments of a single giant planet.

In situ exploration of the giant planets is the only way to completely characterize giant planet compositions in the solar system. In this context, a Saturn probe is the next natural step beyond Galileo's in situ exploration of Jupiter [32], the remote investigation of Jupiter's interior and gravity field by the Juno mission, and the Cassini spacecraft's orbital reconnaissance of Saturn.

\subsection{Measurement Priorities}

An entry probe should reveal new insights into the vertical structures of temperatures, density, chemical composition and clouds during descent through a number of different atmospheric regions, from the stable upper/middle atmosphere to the convective troposphere. The probe would directly sample the condensation cloud decks and ubiquitous hazes whose composition, altitude and structure remain ambiguous due to the inherent difficulties with remote sensing. In addition to bringing fundamental constraints on Saturn's formation conditions, in situ measurements would show how Saturn's atmosphere flows at a variety of different depths above, within and below the condensate clouds. The depth of probe penetration determines whether it can access the well-mixed regions for key condensable volatiles. In the present case, a shallow probe penetrating down to $\sim 10$ bar would in principle sample $\mathrm{NH}_{3}$ and $\mathrm{H}_{2} \mathrm{~S}$ both within and below their cloud bases, in the well-mixed regions of the atmosphere to determine the $\mathrm{N} / \mathrm{H}$ and $\mathrm{S} / \mathrm{H}$ ratios, in addition to noble gases and isotopic ratios. Note that the $\mathrm{N}$ determination could be a lower limit be- 
cause ammonia is highly soluble in liquid water. Also, because the hypothesized water cloud is deeper than at least $\sim 12.6$ bar in Saturn [7], the prospect of reaching the deep $\mathrm{O} / \mathrm{H}$ ratio remains unlikely even if the probe survives beyond its design limit, unless a precise determination of the $\mathrm{CO}$ abundance (or any other species limited by reactions with the tropospheric water) is used to constrain $\mathrm{H}_{2} \mathrm{O} / \mathrm{H}_{2}$. Nevertheless, measuring elemental abundances (in particular $\mathrm{He}$, noble gases and other cosmogenically-common species) and isotopic ratios using a shallow entry probe on Saturn will provide a vital comparison to Galileo's measurements of Jupiter, and a crucial "ground-truth" for the remote sensing investigations by the Cassini spacecraft. Table 1 ranks in order of priority the key in situ measurements that should be carried out by the Hera probe and its associated carrier.

\subsection{Required Instruments}

The scientific requirements discussed above are addressed with a suite of scientific instruments located on the probe or the carrier spacecraft as detailed in Table 2. At minimum, the science payload must contain two core instruments: a Mass Spectrometer (MS) and an Atmospheric Structure Instrument (ASI). These two instruments are sufficient to cover both Priority 1 and Priority 2 measurements. The MS will provide key measurements of the chemical and isotopic composition of Saturn's atmosphere, as well as its mean molecular weight. The key in situ measurements performed by the ASI will be the accelerometry during the probe entry phase and pressure, temperature and density profile during descent. A Radio Science Experiment (RSE), a Nephelometer, a Net flux Radiometer (NFR) and a camera will address Priority 3 measurements. The RSE will include a Doppler Wind Experiment (DWE) dedicated to the measurement of the vertical profile of the zonal (east-west) winds along the probe descent path. It will also include an element dedicated to absorption measurements (AAbs), to indirectly infer the abundance of ammonia. The Nephelometer will be devoted to the investigation of the composition and precise location of cloud layers. The NFR will measure the thermal profile and radiative energy balance in the atmosphere. A camera located on the carrier will provide i) contextual imaging of the probe entry site and ii) global characterization of Saturn's atmosphere at the time of probe entry. The Science Traceability Matrix is represented in Table 3.

\subsection{Probe Entry Zone}

In the present paper, the trajectory selection is based on the selected carrier option, launch vehicle (Soyuz) capabilities, and the available probe thermal protection capability. The interplanetary trajectory and the probe entry location are inseparably linked. Saturn's extensive ring system presents a severe collision hazard to an in- 
bound probe. For various declinations of the spacecraft's approach asymptote, some latitudes are inaccessible because the trajectories to deliver to those latitudes would impact the rings. Also, although it is possible to adjust the inclination of the approach orbit for purposes of accessing desired latitudes, this approach can greatly increase the atmosphere-relative entry speeds, possibly driving the mission to an expensive heat shield material technology development (see Sec. 4). During the ESA assessment study, the issues of probe entry locations, approach and entry trajectories, and probe technologies will have to be treated together.

With a single entry probe, the selected entry site must be carefully studied. Saturn's equatorial zone is one potential site from the scientific point of view for a single entry probe because of its meteorological activity that combines the emergence of large-scale storms [37], vertical wind shears in the troposphere [21], and upwelling enhanced volatiles and disequilibrium species $[14,15]$. However, this may not be typical of Saturn's atmosphere, so detailed trades would need to be discussed during the study phase. Eastward jets (particularly the anticyclonic branch of eastward jets) located at equator might be good locations to retrieve the deep values of volatiles at higher levels in the atmosphere [35]. A primary requirement is that volatile-depleted regions must be avoided for the entry site. These zones are probably located at the cyclones in both poles and may also be located at the so-called "storm-alley" (mid-latitude regions of low static stability able to develop updrafts and downdrafts). In any case, there are several potential entry points and a decision where to enter must also be guided by the design of the thermal protection system of the probe. Envisaging in situ measurements in the equatorial region of Saturn appears to be the best compromise between science and engineering.

\section{Proposed Science Instruments}

\section{Priority}

The Hera Saturn Probe mission will conduct in situ measurements of the structure, composition and fundamental processes operating within Saturn's atmosphere. Measurements will be made by a suite of instruments on the probe as it descends for up to 75-90 minutes under a parachute from the tropopause near $100 \mathrm{mbar}$, through the upper cloud decks, down to at least 10 bars. The Tier 1 instruments, designed to address the highest priority science goals, include a Mass Spectrometer and an Atmospheric Structure Instrument. The instruments comprising the Tier 2 payload address lower priority science goals, and include a Net Flux Radiometer, a Nephelometer, and a Radio Science experiment. While all instruments are located on the Hera probe itself, one ultrastable oscillator for the Radio Science experiment will be mounted on the Carrier. The Carrier will also have a camera operating at visible wavelengths to provide contextual imaging of the probe's entry site and a global characterization of Saturn's atmosphere at the entry time.

All instruments can operate on both the day and night side of Saturn, although the visible channel of the Net Flux Radiometer can only measure the altitude profile 
of solar energy absorption if the descent is on the dayside. The following section provides the investigation and measurement objectives of each instrument, including the measurement principle, the description of the instrument design, the resource requirements including mass, power, volume, and data rate, interface and calibration requirements, and a summary of technology readiness, heritage, and critical issues (if any). The total data returned from the probe will range from 30 to 40 megabits total. Table 4 summarizes the main characteristics of the instruments (size, mass, power requirement, data rate and volume).

\subsection{Hera Mass Spectrometer}

\subsubsection{Investigation Overview}

The chemical, elemental, and isotopic composition of Saturn's atmosphere and its profile down to the 10 bar level will give important clues about the solar nebula at the location of Saturn's formation, about the formation of giant planets (in comparison to Jupiter) and Saturn's evolution to present state. Also, measurement of the composition of Saturn's atmosphere will provide details of the chemical structure of the atmosphere over the descent trajectory, which is not accessible to remote sensing investigations.

The measurement objective of the Hera MS is to provide in situ measurements of the chemical, elemental, and isotopic composition of Saturn's atmosphere, and dependence of composition on pressure/altitude along the descent trajectory of the entry probe. The primary objective is the determination of the abundances of the major chemical species $\mathrm{CH}_{4}, \mathrm{NH}_{3}, \mathrm{H}_{2} \mathrm{O}, \mathrm{H}_{2} \mathrm{~S}$, the $\mathrm{He} / \mathrm{H}$ ratio, and the abundance of the noble gases $\mathrm{Ne}, \mathrm{Ar}, \mathrm{Kr}$ and $\mathrm{Xe}$. Secondary objectives include isotopic ratios of major elements like $\mathrm{H}, \mathrm{He}, \mathrm{C}$, and $\mathrm{N}$, the abundances of minor chemical species and the isotopic abundances of noble gases. Tertiary objective are the abundance of the oxygen isotopes.

\subsubsection{Measurement Principle}

The core of the Hera MS is a time-of-flight mass spectrometer (TOF-MS). Such instruments have several advantages for space research: i) all masses are measured at the same time, thus there is no need for scanning the mass, leading to an increase of the sensitivity, ii) they are simple and robust instruments very suitable for remote operation on a spacecraft, iii) they can be built light-weight. The cadence of mass spectrometric measurements is variable, from mass spectra accumulated every 1-second to integration up to 300 seconds. At suitable times, measurements of atmospheric gas are replaced by measurements of calibration gas, and measurements of gas enriched and separated from the bulk atmosphere. 
The atmospheric gas will enter the experiment via a gas inlet system comprising several independent entrances of various conductances, which will cover the pressure range of 0.1-10 bar level. The cadence of mass spectra is adjusted such that the vertical resolution is about $1.8 \mathrm{~km}$ along descent trajectory, which amounts to a total of about 400 mass spectra along the descent trajectory.

Not all gases can be measured directly in the gas entering from the atmosphere, at least not with the desired accuracy. Noble gases, for example, will be separated from the entering gas and collected by a cryotrap enrichment system. After sufficient enrichment of the noble gases is accomplished they are released to the TOF-MS for a dedicated mass spectrometric measurement while the direct sampling of the atmosphere is interrupted. Similarly, the use of an additional cryotrap for the enrichment of hydrocarbons and other trace species will also be analyzed at regular intervals.

The accuracy of some composition measurements will be enhanced by carrying several reservoirs of reference gases with an accurately known gas mixture. For example, for the measurement of the $\mathrm{He} / \mathrm{H}$ ratio a gas container with a calibrated $\mathrm{He} / \mathrm{H}$ mixture is part of the Hera MS experiment that will allow for the measurement of this ratio with an accuracy of $2 \%$ or better. Similarly, a container with a calibrated mixture of noble gases, and a container with reference gases for key isotopes $(\mathrm{H}, \mathrm{C}$, $\mathrm{N}$, and $\mathrm{O}$ ), are included in the Hera Mass Spectrometer.

\subsubsection{Design Description / Operating Principle}

The Hera Mass Spectrometer consists of four units: the TOF-MS, the Tunable Laser Spectrometer (TLS), the gas separation and enrichment system (GSES), and the reference gas system (RGS).

The TOF-MS consists of a pulsed ion source, a time-of-flight drift path, an ion mirror (reflectron), and a fast ion detector. The TOF-MS is a compact instrument and has a mass range of 1-1000 u/e, a mass resolution of $m / \Delta m=1100$, and a very high sensitivity [47]. Ions, continuously generated in the ion source, are pulseextracted, and sent as ion packets along the TOF path with a repetition frequency of $10 \mathrm{kHz}$, to the detector resulting in a mass spectrum. These spectra are accumulated for a defined integration period (1-300 seconds), depending on the desired vertical resolution along the descent trajectory. The integration of many spectra provides for a dynamic range of 6-7 decades in each accumulated spectrum; together with various detector gain steps and the gas enrichments at a dynamic range that exceeds 12 decades is achieved.

The Tunable Laser Spectrometer (TLS) [13] will be employed as part of the Hera MS to measure the isotopic ratios with high accuracy of the molecules $\mathrm{H}_{2} \mathrm{O}, \mathrm{NH}_{3}$, $\mathrm{CH}_{4}, \mathrm{CO}_{2}$ and others. TLS employs ultra-high spectral resolution tunable laser absorption spectroscopy $\left(\Delta v=0.0005 \mathrm{~cm}^{-1}\right)$ in the near infra-red (IR) to mid-IR spectral region. TLS is a direct, non-invasive, and simple technique that for small mass and volume achieves sensitivities at the sub-ppb level for gas detection. Species abundances can be measured with accuracies of a few percent, and isotope determinations have an accuracy of about $0.1 \%$. With the TLS system one can derive 
the isotopic ratios of $\mathrm{D} / \mathrm{H},{ }^{13} \mathrm{C} /{ }^{12} \mathrm{C},{ }^{15} \mathrm{~N} /{ }^{14} \mathrm{~N},{ }^{18} \mathrm{O} /{ }^{16} \mathrm{O}$, and ${ }^{17} \mathrm{O} /{ }^{16} \mathrm{O}$. A recent use of a TLS system was in the Sample Analysis at Mars (SAM) GC-MS system on Mars Science Laboratory (MSL) [45, 26]. The GSES consists of a cryotrap, an ion pump, and a non-evaporable getter (NEG), which together are used to achieve the noble gas enhancement. The NEG removes all constituents except methane and the noble gases. The cryotrap traps the products of the NEG process, except for helium and some neon. The ion pump then operates to pump away the helium, which is the second most abundant species in Saturn's atmosphere, thus enhancing the signal to noise ratio in the ${ }^{17} \mathrm{O} /{ }^{16} \mathrm{O}$ remaining noble gases by about 200 times. This enrichment cell will be accessed periodically during descent to allow the noble gases to be analysed. The cryotrap for minor species will have a separate gas inlet. It will be heated periodically and a valve opened to allow the descent measurements to be interrupted for analysis.

The Reference Gas System consists of a central manifold and pressure sensor connected to the mass spectrometer via a capillary leak. Reference gas mixtures are stored in stainless steel $1 \mathrm{ml}$ containers at a pressure of approximately 1 bar. Each reference gas will be admitted into the manifold by opening a single valve in a short

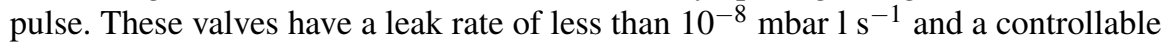
pulse width of less than $1 \mathrm{~ms}$; they are a development from Ptolemy Rosetta heritage (TRL 5). Alternative valves are the same as used on Philae (TRL 9) but have a higher power requirement and a longer operating cycle of several minutes.

The baseline proposal includes 3 reference gas mixtures; a hydrogen/helium mixture, a noble gas mixture and an isotope mixture. The composition of the isotopic reference gas will be a mixture of relatively inactive molecules, e.g. methane, carbon monoxide and nitrogen, depending upon the scientific targets.

The RGS includes an ion pump and non-evaporable getter to remove gases between analyses and allow calibration of the mass spectrometer during cruise, a few hours before atmospheric entry and during the atmospheric descent. The ion pump adds a significant mass to the RGS, which could be reduced by using the GSES pump instead; however this adds to the complexity in the timing between the two systems and potentially results in cross contamination between the reference gases and the atmospheric samples (see Fig. 1).

\subsubsection{Mass, Power, Data Rate}

Mass and power of the MS elements are summarized in Table 5. The average data rate during descent is about $2 \mathrm{kbit} / \mathrm{s}$ (including $50 \mathrm{bit} / \mathrm{s}$ housekeeping data). The total data volume produced during a 90-minute descent is about 10.7 Mbit of compressed data. 


\subsubsection{Specific/Critical Interface Requirements \& Environment Constraints}

The Hera Mass Spectrometer has to be mounted on the entry probe such that the gas sampling entrance is in the atmospheric gas flow during descent. No chemical contamination from the entry probe should enter the gas sampling system during descent. Otherwise the Hera MS is accommodated inside the entry probe in a stable thermal environment. There are no special requirements for radiation and magnetic cleanliness.

The Hera MS should be turned on before starting the scientific measurements to execute a warm-up procedure to reach stable operating conditions, including background measurements, instrument optimisation and calibration in time for the scientific operations. The exact turn-on procedure will be defined during phase A when more technical details of the entry probe are available. For the direct atmospheric sampling, the GSES, and the TLS and gas chromatograph share a gas flow line with a gas inlet port in the entry probe fore dome at the apex near the stagnation point and an outlet port at the minimum pressure point at the rear of the probe. Metal ceramic devices seal the inlet and outlet ports. The inlet and outlet lines are evacuated after instrument calibration prior to shipment, and will be opened in sequence by redundant pyrotechnic actuators after probe entry and ejection of the probe front shield.

\subsubsection{Specific Calibration Requirements}

The Hera MS will be thoroughly calibrated before delivery for entry probe integration. Before starting scientific operations during descent the Hera MS will execute an automatic optimisation programme to define the instrument operation parameters. This is followed by a calibration programme using the reference gases. A replica reference gas system will be stored in similar environmental conditions to the entry probe to monitor any changes during the long cruise. This is particularly important for the hydrogen/helium mixture as both hydrogen and helium will diffuse through the walls of the stainless steel container. Because of the changing temperature environment during descent, calibration using the RGS will be repeated several times during the descent.

\subsubsection{TRL Assessment \& Relevant Heritage}

There is plenty of heritage for measuring the chemical composition during a descent through the atmosphere, for example the Galileo Probe mass spectrometer system [30] or the Huygens Gas Chromatograph mass spectrometer [29]. Over the last two decades TOF mass spectrometers have been developed for space research, for example on the Rosetta mission [8], which have several advantages over the quadrupole mass spectrometers used before. For example, a TOF-MS is over 1000 times more sensitive than the Cassini INMS (ten times from ion source efficiency and 100 times 
from better duty cycle). Also for the gas inlet system and the gas enrichment system there is plenty of heritage from previous missions, again the Galileo Probe mass spectrometer system, the Huygens Gas Chromatograph mass spectrometer, or more recently the mass spectrometer experiments on the Rosetta lander.

During phase A, a trade study will be conducted where the selected solution of having a high-sensitivity mass spectrometer with medium mass resolution together with a TLS instrument for selected isotope measurements will be compared to a single sensitive high-resolution mass spectrometer. The latter would allow all measurements to be performed with a single instrument, at the price of increased complexity of the instrument.

\subsubsection{Critical Issues}

None.

\subsection{Hera Atmospheric Structure Instrument}

\subsubsection{Investigation overview}

The Hera Atmospheric Structure Instrument (Hera-ASI) will make in situ measurements during entry and descent into the Saturn's atmosphere in order to investigate the atmospheric structure and dynamics, and electricity. The Hera-ASI scientific objectives are the determination of the atmospheric vertical pressure and temperature profiles, the evaluation of the density along the Probe trajectory and the investigation of the atmospheric electricity (e.g. lightning) by in situ measurements. Hera-ASI data will also contribute to the analysis of the atmospheric composition. Moreover, Hera-ASI will have a primary engineering function by establishing the entry trajectory and the probe altitude and vertical velocity profile for correlating all probe experiment data and to support the analysis of the Radio Science / Doppler Wind Experiment (DWE).

In situ measurements are essential for the investigation of the atmospheric structure and dynamics. Hera ASI will measure the atmospheric state $(P, T$ and density) as well as constraining atmospheric stability and dynamics, and the effect on atmospheric chemistry. The estimation of the temperature lapse rate can be used to identify the presence of condensation and eventually clouds, and to distinguish between saturated and unsaturated, stable and conditionally stable regions. The vertical variations in the density, pressure and temperature profiles provide information on the atmospheric stability and stratification, on the presence of winds, thermal tides, waves and turbulence in the atmosphere.

Hera ASI will measure properties of Saturn lightning, determine the conductivity profile of the Saturnian troposphere, and detect the atmospheric DC electric field. It is well known that $2000-\mathrm{km}$ sized atmospheric storm systems on Saturn produce 
superbolt-like lightning discharges with energies up to $10^{13} \mathrm{~J}$. To date the strong Saturn lightning radio emissions have only been measured from outside Saturn's ionosphere, i.e. mostly at frequencies $>1 \mathrm{MHz}$ and occasionally down to a few hundred kHz. Hence Hera ASI will measure the unknown lightning spectrum in the frequency range of $\sim 1-200 \mathrm{kHz}$, and obtain burst waveforms with different temporal resolutions and durations. A Saturn lightning flash typically lasts $\sim 100$ $\mathrm{ms}$ and consists of many sub-discharges of the order of $0.1 \mathrm{~ms}$, so waveforms over $100 \mathrm{~ms}$ with $0.1 \mathrm{~ms}$ resolution for the full flash and waveforms over $0.5 \mathrm{~ms}$ with $2 \mu \mathrm{s}$ resolution for the sub-strokes would be a sensible choice. The latter requires a sampling frequency of $500 \mathrm{kHz}$, which is also sufficient for obtaining the spectrum up to $200 \mathrm{kHz}$.

Atmospheric conductivity and the DC electric field are important basic parameters of atmospheric electricity which provide indirect information about galactic cosmic ray ionization, aerosol charging inside and outside of clouds, properties of potential Schumann resonances and so on.

\subsubsection{Measurement Principle}

The key in situ measurements will be atmospheric density, pressure and temperature profile by measuring deceleration of the entry vehicle and performing direct temperature and pressure measurements during the descent phase [19, 38]. Densities will be determined using measurements of the deceleration of the probe during entry. The flight profile of the probe, including variations in speed and angle of attack provide information regarding turbulence and vertical motions. Once the probe heat shield is jettisoned, direct measurements of pressure, temperature and electrical properties will be performed. Hera ASI will monitor the acceleration experienced by the probe during the whole descent phase and will provide the unique direct measurements of pressure, temperature, conductivity, and DC electric field through sensors having access to the atmospheric flow.

\subsubsection{Design Description / Operating Principle}

The Hera Atmospheric Structure Instrument (ASI) consists of several sensors both internal and external to the pressure vessel, and operates during high speed entry in the upper atmosphere and in descent when the probe is subsonic. The proposed instrument design leverages strongly from the Huygens ASI experiment of the Cassini/Huygens mission [20] and the Galileo and Pioneer Venus ASI instruments [39, 40]. The Hera ASI consists of four primary sensor packages: (i) a three axial accelerometer (ASI-ACC), (ii) a pressure profile instrument (ASI-PPI), (ii) temperature sensors (ASI-TEM) and (iv) an Atmospheric Electricity Package (ASIAEP). The control, sampling and data management of the ASI sensors is handled by a central Data Processing Unit including the main electronics for the power supply 
and conditioning, input/output and sensor control. The ASI-DPU interfaces directly to the entry probe processor.

The ASI-ACC will start to operate prior to the beginning of the entry phase, sensing the atmospheric drag experienced by the entry vehicle. Direct pressure and temperature measurement will be performed by the sensors having access to the atmospheric flow from the earliest portion of the descent until the end of the probe mission at approximately 10 bars. AEP will measure the atmospheric conductivity and DC Electric field in order to investigate the atmospheric electricity and detecting lighting.

\section{Accelerometers}

The ACC package consisting of 3-axis accelerometers should be placed as close as possible to the center of mass of the entry vehicle. The main sensor is a highly sensitive servo accelerometer aligned along the vertical axis of the Probe, with a resolution of $10^{-5}$ to $10^{-4} \mathrm{~m} / \mathrm{s}^{2}$ (depending on the resolution setting) with an accuracy of $1 \%$. Accelerations can be measured in the $0-200 \mathrm{~g}$ range (where $\mathrm{g}$ is the Earth's acceleration of gravity). The Huygens servo accelerometer is the most sensitive accelerometer ever flown in a planetary entry probe [48]. Having a triaxial accelerometer (namely one sensor located along each probe axis) will allow for an accurate reconstruction of the trajectory and attitude of the probe, and to sense the atmospheric drag in order to derive the entry atmospheric density profile. Assuming the HASI ACC Servo performance at Titan, a noise performance of some $0.3 \mu \mathrm{g}$ is expected. The exact performance achievable, in terms of the accuracy of the derived atmospheric density, will also depend on the probe ballistic coefficients, entry speed and drag coefficient, all of which will differ somewhat from the Titan case.

\section{Pressure Profile Instrument}

The ASI-PPI will measure the pressure during the entire descent with an accuracy of $1 \%$ and a resolution of 1 micro bar. The atmospheric flow is conveyed through a Kiel probe inside the Probe where the transducers and related electronic are located.

The transducers are silicon capacitive sensors with pressure dependant dielectricum. The pressure sensor contains a small vacuum chamber as dielectricum between the two electrode plates, where the external pressure defines the distance of these plates. Detectors with diaphragms of different pressure sensitivity will be utilized to cover the pressure range to $\sim 10$ bar. The pressure is derived as a frequency measurement (within 3-20 kHz range) and the measurements is internally compensate for thermal and radiation influences. 


\section{Temperature Sensors}

The Temperature Sensors (TEM) utilize platinum resistance thermometers to measure the kinetic temperature during the descent just as in the Huygens Probe ASI and Galileo probe. Two thermometers are exposed to the atmospheric flow and effectively thermally isolated from the support structure. Each thermometer includes two redundant sensing elements: the primary sensor (FINE) directly exposed to the airflow and a secondary sensor embedded into the supporting frame with the purpose to be used as spare unit in case of damage of the primary. The principle of measurement is based on the variation of the resistance of the metallic wire with temperature. The reading of the thermometer is made by resistance comparison with a reference resistor, powered by a pulsed current.

TEM has been designed in order to have a good thermal coupling between the sensor and the atmosphere and to achieve high accuracy and resolution. Over the temperature range of $60-330 \mathrm{~K}$ these sensors maintain an accuracy of $0.1 \mathrm{~K}$ with a resolution of $0.02 \mathrm{~K}$.

\section{Atmospheric Electricity Package}

The Atmospheric Electricity Package (AEP) consists of sensors and a signal processing unit. Since Saturn's lightning is very intense and localized, it should be detectable by a short electric monopole, dipole, loop antenna or double probe from distances of several thousands of kilometers. The conductivity of the atmosphere can be measured with a mutual impedance probe. A current pulse is sent through the surrounding medium and the resulting voltage is measured by two passive electrodes from which the impedance of the medium can be determined. This can be corroborated by determining the discharge time (relaxation) of two charged electrodes. After the discharge, the natural DC electric field around the probe can also be measured with them. The signal processing unit (to be accomodated into the ASI main central unit) will amplify the signals, extract waveforms of bursts with different durations and temporal resolutions, perform spectral analysis at various frequency ranges $(1-200 \mathrm{kHz}$ or in the TLF below $3 \mathrm{~Hz}$ to detect Schumann resonances), and to provide active pulses and sensor potential control to handle the conductivity and DC electric field measurements.

\subsubsection{Mass, Power, Data Rate}

The required resources of the Hera-ASI are based on estimates made from the heritage of the Huygens Atmospheric Structure Instrument [20].

- Mass: $\sim 1.5 \mathrm{~kg}$ (including sensors, electronics, and supporting structure/boom);

- Volume: $20 \times 20 \times 20 \mathrm{~cm}^{3}$ (distributed, including DPU);

- Data Rate: 250 bps (without compression); 
- Power: $\sim 10$ watts.

It is expected that the performance and requirements will be improved based on experience from the Huygens ASI, in terms of sensor design, packaging, and mounting, as well as the benefit of nearly two decades of technological improvements in sensor technology.

\subsubsection{Specific/Critical Interface Requirements \& Environment Constraints}

The accelerometer package should be mounted as close as possible to the probe's center of mass. The TEM temperature sensors and the PPI pressure Kiel inlet should be mounted onto a fixed external sensor boom ensuring that the sensors are outside of the probe boundary layer. Electric sensors should be accommodated on a boom in order to measure the DC electric field in vertical direction. EMC requirements and ESD protection have to be taken into account. define EMC and ESD

Heat shield Thermal Protection System (TPS) sensors, like those for MSL MEDLI and ESA ExoMars, will provide important data on TPS mass loss and heat shield dynamics during entry. These type of measurements proved to be critical for the Galileo entry probe [27].

\subsubsection{Specific Calibration Requirements}

Static and dynamical calibration will be performed at sensor, subsystem and instrument integrated level. Stratospheric balloon drop test experiments with the ASI package could be useful in order access sensors performance and to validate data retrieval methods. During cruise phase, CheckOuts $(\mathrm{CO})$ will be regularly performed, ASI sensor performance will be monitored in order to check any drift due to aging or any degradation. Specifically on Huygens, in-flight data have been used in order to monitor the offset at zero $g$ of the ACC sensor and estimate the long-term stability in the zero offset [48].

\subsubsection{TRL Assessement \& Relevant Heritage}

Each Hera-ASI component has strong application heritage tracking back through Pioneer Venus, and Galileo ASI instruments [39, 40] and Huygens ASI experiment of the Cassini/Huygens mission [20].

The Huygens ASI ACC main servo sensor is the most sensitive accelerometer ever flown in a planetary entry probe [48]. Having a triaxial accelerometer (namely one sensor located along each probe axis) will allow for an accurate reconstruction of the trajectory and attitude of the probe, and to sense the atmospheric drag in order to derive the atmospheric density profile.

The TEM utilizes platinum resistance thermometers just as in the Huygens Probe ASI, and Galileo and Pioneer Venus probes. The proposed type of the pressure 
sensors, other than being successfully flown as part of HASI on board the Huygens probe, have been flown on the NASA's Mars Phoenix 2007 mission and Mars Science Laboratory (MSL) and are selected as part of the meteorological package of ESA's ExoMars 2016 EDM mission (DREAMS) and NASA's Mars 2020 rover (MEDA).

The Galileo Probe LRD (Lightning and Radio emission Detector) used a ferritecore RF antenna and two photodiodes behind lenses to measure magnetic field pulses and optical emissions of lightning. Since Saturn lightning storms are quite localized (e.g. in the storm alleys at $\pm 35^{\circ}$ latitude) and might not be present all the time, we prefer not to use an optical detector, but rather employ an instrument design similar to the Huygens Probe HASI- PWA (Permittivity, Waves and Altimetry) Analyzer [23] including measurements of atmospheric electrical conductivity and the DC electric field.

In the Hera ASI design, existing flight-proven or commercial, off-the-shelf (COTS) hardware is applied in proven processes and applications. Other possible sensors types/candidates could be evaluated during the Phase A, but all of the components in the Hera ASI are at TRL higher than 6.

\subsubsection{Critical Issues}

None

\subsection{Hera Net Flux Radiometer Experiment}

\subsubsection{Investigation Overview}

Two notable NFR instruments have flown in the past namely, the Large Probe Infrared Radiometer (LIR) [9] on the Venus Probe, and the Net Flux Radiometer (NFR) on the Galileo Probe [43] for in situ measurements within Venus and Jupiter's atmospheres, respectively. Both instruments were designed to measure the net radiation flux and upward radiation flux within their respective atmospheres as the Probe descended by parachute. The NASA GSFC Net Flux Radiometer (see Fig. 2), builds on the lessons learned from the Galileo Probe NFR experiment and is designed to determine the net radiation flux within Saturn's atmosphere. The nominal measurement regime for the NFR extends from $\sim 0.1$ bar to at least 10 bars, corresponding to an altitude range of $\sim 79 \mathrm{~km}$ above the 1 bar level to $\sim 154 \mathrm{~km}$ below it. These measurements will help to define sources and sinks of planetary radiation, regions of solar energy deposition, and provide constraints on atmospheric composition and cloud layers.

The primary objective of the NFR is to measure upward and downward radiative fluxes to determine the radiative heating (cooling) component of the atmospheric energy budget, determine total atmospheric opacity, identify the location of cloud 
layers and opacities, and identify key atmospheric absorbers such as methane, ammonia, and water vapor. The NFR measures upward and downward flux densities in two spectral channels. The specific objectives of each channel are:

- Channel 1 (solar, 0.4-to-5 $\mu \mathrm{m}$ ). Net flux measurements will determine the solar energy deposition profile; upward flux measurements will yield information about cloud particle absorption and scattering;

- Channel 2 (thermal, 4-to-50 $\mu \mathrm{m}$ ). Net flux measurements will define sources and sinks of planetary radiation. When used with calculations of gas opacity effects, these observations will define the thermal opacity of particles.

\subsubsection{Measurement Principle}

The NFR measures upward and downward radiation flux in a $5^{\circ}$ field-of-view at five distinct look angles, i.e., $\pm 80^{\circ}, \pm 45^{\circ}$, and $0^{\circ}$, relative to zenith/nadir. The radiance is sampled at each angle approximately once every $\sim 2 \mathrm{~s}$.

The NFR Focal Plane Assembly (FPA), Figure 3, is comprised of bandpass filters, folding mirrors, non-imaging Winston cone concentrators, and radiation hard uncooled thermopile detectors housed in a windowed vacuum micro-vessel that is rotated to the look angle by a stepper motor. NASA GSFC has been working on this approach to develop a Saturn Probe NFR for a few years and has extensive experience with the detectors and electronics.

Assuming a thermopile voltage responsivity of $295 \mathrm{~V} / \mathrm{W}$, an optical efficiency of $50 \%$, a detector noise of $18 \mathrm{nV} / \sqrt{\mathrm{Hz}}$ and an ASIC input referred noise of 50 $\mathrm{nV} / \sqrt{\mathrm{Hz}}$ with 12-bit digitization gives a system signal-to-noise ratio of 300 to 470 in the solar spectral channel and 100 to 12800 in the thermal spectral channel for atmospheric temperature and pressure ranges encountered in the descent, i.e., 80 to $300 \mathrm{~K}$ and 0.1 to 10 bar respectively.

\subsubsection{Design Description / Operating Principle}

A physical and functional block diagram of the NFR is shown in Fig. 4. The focal plane consists of four single pixel thermopile detectors (solar, thermal and two dark channels), bandpass filters and Winston concentrators. The Front End Electronics (FEE) readout, see inset of Fig. 5, uses a custom radiation-hardened-bydesign mixed-signal ASIC for operation with immunity to $174 \mathrm{MeV}-\mathrm{cm}^{2} / \mathrm{mg}$ single event latch-up and $50 \mathrm{Mrad}$ (Si) total ionizing dose [33]. The ASIC has sixteen lownoise chopper stabilized amplifier channels that have configurable gain/filtering and two temperature sensor channels that multiplex into an on-chip 16-bit sigma-delta analog-digital converter (SDADC). The ASIC uses a single input clock $(\sim 1.0 \mathrm{MHz})$ to generate all on-chip control signals such as the chopper/decimation clocks and integrator time constants. The ASIC also contains a radiation tolerant 16-bit $20 \mathrm{MHz}$ Nyquist ADC for general-purpose instrumentation needs. 
The Main Electronic Box (MEB) is a redundant electrical system for science and housekeeping telemetry and thermal sensing and control. The two main elements of the MEB are the instrument and motor control board (comprising the instrument control and the motor drive electronics) and the Low Voltage Power Supply (LVPS) board.

The instrument control electronics uses a radiation hard $\mu$-processor (e.g., Intersil HS-80C85RH) to perform the following functions: (i) receive and process the serial digitized data from the thermopile channels as well as provide a master clock and tagged encoded commands to the ASIC command decoders via a single line; (ii) mathematical operations on the science data such as averaging or offset corrections; (iii) data reduction, packetization, and routing of the science and housekeeping data to the Probe via a RS422 protocol; (iv) receive and act upon commands received from the Probe, e.g., active channel selection, setting temperature levels, or motor positions; (v) control stepper motor positions as well as decode their respective positions; (vi) provide stable temperature control to the instrument; and (vii) collect all temperatures and supply and reference voltages to form housekeeping/time stamped header packets that are streamlined into the data output to the Probe. All timing functions are synchronized with a 1 pulse per second (PPS) square wave from the Probe. The LVPS board accommodates DC-DC convertors and other various voltage/current control devices. This board not only conditions and regulates the voltages for various electronic usage but also controls power to the heaters. The Probe +28 VDC bus voltage is filtered and dropped via DC-DC switch mode converter to two main voltages: +3.3 VDC for logic use and +5 VDC for the stepper motor.

\subsubsection{Volume, Mass, Power, Data Rate}

- Mass: $2.4 \mathrm{~kg}$ (including harness and 5\% margin);

- Volume: $\sim 113 \mathrm{~mm} \times 144 \mathrm{~mm} \times 279 \mathrm{~mm}$;

- Power: $\sim 5 \mathrm{~W}$;

- Data Rate: $\sim 55$ bps (average);

- Data Volume: 297 Kbits (90-minutes).

\subsubsection{Specific/Critical Interface Requirements \& Environment Constraints}

The NFR will be mechanically mounted using thermally isolating mounts onto the Probe deck so accurate knowledge of the deck temperature, to better than $\pm 1 \mathrm{~K}$, is required. An additional +28 VDC supply is needed for survival heaters that are controlled from the Probe.

The power-on for thermal control of the instrument must also be carefully considered prior to deploying the Probe from the carrier relay spacecraft. The heaters may require turning on at least $36-48$ hours prior to the Probe telemetry. Once the 
parachute is deployed at the top of the atmosphere, the descent latitude/longitude should be known to $\pm 0.1^{\circ}$, the drop and spin rate should be known to $\pm 0.1 \mathrm{~m} / \mathrm{s}$.

\subsubsection{Specific Calibration Needs}

The NFR will be radiometrically calibrated, in a thermal-vacuum chamber that simulates the environmental conditions prior to launch to establish both offset and gain uncertainties as a function of temperature. The gain uncertainity during descent is calibrated and removed by performing views of the on-board hot and cold calibration targets as the instrument cycles sequentially through the look angles.

\subsubsection{TRL and Relevant Heritage}

The TRL level for all components and subsystems in the NFR is $>6$. NASA GSFC has decades of experience managing, designing and delivering planetary mission instruments like the NFR.

\subsubsection{Critical Issues: None}

\subsection{Hera Probe Nephelometer}

\subsubsection{Investigation Overview}

Knowing the micro- and macro-physical properties of the haze and cloud particles in Saturn's atmosphere is crucial for understanding the chemical, thermo-dynamical and radiative processes that take place. Full characterization of the various types of haze and cloud particles requires in situ instrumentation, because Saturn's stratospheric hazes obscure the lower atmosphere, and because remote-sensing measurements of (for example) reflected sunlight depend on myriads of atmospheric parameters thus prohibiting reaching unique solutions. The Hera Nephelometer (NEPH) will illuminate haze and cloud particles, and will measure the flux and degree of linear polarization of the light that is scattered in a number of directions. The particle properties can be derived from the dependence of the scattered flux and polarization on both the scattering angle and the wavelength.

The primary measurement objective of the Nephelometer is to characterize the micro- and macro-physical properties of atmospheric particles by measuring the flux and polarization of light that is scattered by particles that are passively sampled along the probe's descent trajectory. The angular and spectral distribution of the flux and polarization of the scattered light provides the particles' size distribution, composition, and shape, as well as their number density. NEPH's secondary objective is to measure the flux and polarization of diffuse sunlight in the atmosphere. This 
will provide the optical depth along the trajectory and its spectral variation, placing the results on the samples into a broad perspective. Combining NEPH's results with ASI's ambient pressure measurements, the absolute vertical profile of the hazes and clouds along the probe's descent trajectory can be determined.

\subsubsection{Measurement Principles}

NEPH consists of two modules: LOAC (Light Optical Aerosol Counter) to measure the size distribution of particles, and PAVO (Polarimetric Aerosol Versatile Observatory) to also measure their shape and composition. The modules will be placed side by side to sample similar particles. The probe's descent through the atmosphere allows LOAC and PAVO to sample particles passively. The low solar flux levels in Saturn's atmosphere require both LOAC and PAVO to use artificial light sources for illuminating their samples.

Figure 6 shows a schematic of LOAC. Sampled particles cross a 2-mm diameter LED light-beam and the flux $F$ of the light that is scattered by a single particle across angle $\Theta=12^{\circ}$ is measured. The scattered flux $F$ is very sensitive to the particle size, but relatively insensitive to its shape and/or composition. LOAC can accurately retrieve particle sizes between 0.1 and $250 \mathrm{~mm}$ in 20 size classes.

Figure 7 shows PAVO's design. PAVO measures flux $F$ as well as degree $P$ and angle $\chi$ of linear polarization [24] of light that is scattered by sampled particles at 9 angles $\Theta: 12^{\circ}$ (the same as for LOAC), $30^{\circ}, 50^{\circ} 70^{\circ}, 90^{\circ}, 110^{\circ}, 130^{\circ}, 150^{\circ}, 170^{\circ}$. At each $\Theta$, a small optical head (without moving elements) translates the scattered light into two modulated flux spectra $F_{M}$ :

$F_{M}(\Theta, \lambda)=0.5 \mathrm{~F}(\Theta, \lambda)[1 \pm P(\Theta, \lambda) \cos \Psi]$

where $\Psi(\Theta, \lambda)=2 \chi(\Theta, \lambda)+2 \pi \delta / \lambda$, with $\lambda$ the wavelength and $\delta$ the retardance of the optical retarder in the head [41] (see also Keller and Snik, patent application WO2014/111487 A1). The \pm sign in the equation represents the beam-splitter in each head that produces two modulated flux spectra at every $\Theta$ that are subsequently fed to the spectrograph and detector with an optical fibre. Each modulated spectrum provides $P$ and $\chi$, while the sum of two spectra yields $F$. PAVO uses LEDs covering 400 to $800 \mathrm{~nm}$ to illuminate its sampled particles.

An extra optical head at $\Theta=0^{\circ}$ monitors variations of non-scattered LED-light to obtain information on the number of particles. By chopping the incident beam, we will be able to derive the local diffuse solar radiation field. Another, outward pointing optical head could be added to directly measure the diffuse solar flux and its polarization state.

From the modulated spectra $F_{M}$ the scattered fluxes $F$ can be derived with a few nm resolution, and $P$ and $\chi$ with $10-20 \mathrm{~nm}$ resolution. The required accuracy for $P$ is $0.005(0.5 \%)$, well within the modulation technique's accuracy [36]. 


\subsubsection{Volume, Mass, Power, Data Rate}

Table 6 represents the characteristics of LOAC and PAVO. The data rate of PAVO increases linearly with the number of optical heads. On-board data processing will keep the data rate low (instead of transmitting the high-spectral resolution, modulated flux spectra, low resolution continuum flux and polarization data will be transmitted). The mentioned data rates assume continuous measurements. The total data transmission of NEPH will be optimized with the desired vertical resolution and the probe's data rate.

\subsubsection{Specific/Critical Interface Requirements}

The nephelometer must be able to sample atmospheric particles and should thus be located on the outside of the payload, preferably on the lowest part of the probe, to avoid any biasing of the samples due to the flow around the probe.

\subsubsection{Specific Calibration Requirements}

LOAC includes a stray-light correction system to remove diffuse solar flux. The stability of its LED should be characterized pre-launch. PAVO has no requirement on the shape of the LED-spectrum, although it should be known in order to derive the absolute scattered flux $F$. The flux (and polarization) spectrum of the LED will be calibrated before sampling particles, using the dedicated optical head at $\Theta=$ $0^{\circ}$. The components in the optical heads can be chosen such that their temperature sensitivity is minimal [41] and will be calibrated before launch (this calibration can be compared against the in-flight calibration of the $0^{\circ}$-head). The scattered and non-scattered LED-light can be distinguished from diffuse sunlight by chopping the incident beam through switching it on and off. The sensitivity of the LED output to this chopping will be determined before launch. Note that $P$ and $\chi$ are independent of the incident LED-spectrum.

\subsubsection{TRL and Relevant Heritage}

LOAC's design is based on an instrument already in use as balloon payload for aerosol size determination in the Earth's atmosphere [36, 41]. PAVO's optical design is based on the SPEX instrument [36] that is used on the ground to measure aerosol properties. The SPEX-optics has been tested successfully for radiation hardness with view of ESA's JUICE mission. A design similar to PAVO's (except for the polarimetric optical heads) is the nephelometer on the Galileo probe [34]. 


\subsubsection{Critical Issues: None}

\subsection{Hera Probe Radio Science Experiment}

\subsubsection{Investigation Overview}

The Hera Probe Radio Science Experiment will comprise two main elements. Radio tracking of the Hera probe from the Carrier Relay Spacecraft (CRSC) while Hera is under parachute will utilize the resulting Doppler shift to provide a vertical profile of zonal winds along the descent path for the duration of the probe telecommunications link detectability to at least ten bars $[4,5,10]$. The possibility for a measurement of the second horizontal component of the winds via a probe signal frequency measurement on Earth when the probe descends on the sub-solar side of Saturn [16, 18] will be carefully explored. The Radio Science/Doppler Wind Experiment (DWE) utilizes the Hera radio subsystem, knowledge of the Hera probe descent location, speed, and altitude profile, and the CRSC trajectory to make a precise determination of the probe speed relative to the CRSC from which the zonal wind drift can be extracted. Additionally, as the Hera probe is expected to drift by up to several degrees in longitude under the influence of the zonal winds, the reconstruction of the probe descent location will provide an improved geographical context for other probe science investigations.

Additionally, the Radio Science/Atmospheric Absorption Experiment (AAbs) will utilize the Hera radio subsystem mounted on the CRSC to monitor the signal strength of the probe signal, providing a measurement of the integrated atmospheric absorption along the signal propagation path. The Galileo probe used this technique at Jupiter to strongly constrain the atmospheric $\mathrm{NH}_{3}$ profile, complementing the atmospheric composition measurements of the probe Mass Spectrometer [17].

The primary objectives of the Hera Probe Radio Science Experiment are therefore to 1) use the probe radio subsystem (both mounted on the probe and the CRSC) to measure the altitude profile of zonal winds along the probe descent path with an accuracy of better than $1.0 \mathrm{~m} / \mathrm{s}$, and 2) to measure the integrated profile of atmospheric absorption, expected to be primarily due to $\mathrm{NH}_{3}$ between the probe and CRSC. Secondary objectives include the analysis of Doppler modulations and frequency residuals to detect, locate, and characterize regions of atmospheric turbulence, convection, wind shear, and to provide evidence for and characterize atmospheric waves, and from the signal strength measurements, to study the effect of refractive-index fluctuations in Saturn's atmosphere including scintillations and atmospheric turbulence. [3, 17].

\subsubsection{Measurement Principle}

The Hera Transmitter UltraStable Oscillator (TUSO) will generate a stable signal for the probe radio link. The receiver USO (RUSO) will provide very accurate mea- 
surements of the probe link frequency. Knowledge of the probe descent speed and the CRSC trajectory will allow the retrieval of Doppler residuals due to unresolved probe motions including wind. While in terminal descent beneath the parachute, the vertical resolution of the zonal wind measurements will depend upon the probe descent speed [6]. In the upper atmosphere the vertical resolution will be on the order of $7 \mathrm{~km}$, while in the deeper atmosphere variations with a vertical scale size on the order of one $\mathrm{km}$ can be detected. The accuracy of the wind measurement will primarily be limited by the stabilities of the TUSO and RUSO, the reconstruction accuracy of the probe and CRSC trajectories, and the relative geometry of the Hera and CRSC spacecraft. Assuming a UHF link frequency, a wind measurement accuracy better than $0.2 \mathrm{~m} / \mathrm{s}$ is expected $[3,11]$.

\subsubsection{Design Description / Operation Principle}

The Hera probe telecommunications system will consist of the radio transmitter subsystem on the probe and the receiver subsystem on the CRSC. The carrier receiver will be capable of measuring the Hera telemetry frequency at a sampling rate of at least 10 samples per second with a frequency measurement accuracy of $0.1 \mathrm{~Hz}$. The signal strength will be measured with a sample rate of $20 \mathrm{~Hz}$ and a signal strength resolution of $.01 \mathrm{dBm}$ [17]. These sampling rates will enable probe microdynamics such as probe spin and pendulum motion, atmospheric waves, aerodynamic buffeting and atmospheric turbulence at the probe location to be detected and measured.

The long-period stability of both the TUSO and RUSO, defined in terms of 30minute fractional frequency drift, should be no greater than $\Delta f / f=10^{-11}$, with an Allan Deviation (at 100-s integration time) of $\sim 10^{-13}$. The USO drift during the probe descent ( 90 minutes maximum) is $0.01 \mathrm{~Hz}$.

\subsubsection{Volume, Mass, Power, Data Rate}

- Ultrastable Oscillator Mass: $\sim 1.5 \mathrm{~kg}$;

- Ultrastable Oscillator Power: $~ 3 \mathrm{~W}$ steady state (higher during warmup);

- Volume: cylinder, $\sim 4 \mathrm{~cm}$ diam. $\times 14 \mathrm{~cm}$ length;

- Data Rate: Very low. USO temperatures and voltages should be measured several times/minute with a corresponding data rate on the order of 2 bps $[1,2,3]$.

\subsubsection{Specific/Critical Interface Requirements \& Environment Constraints}

To avoid spurious contributions to the Doppler profile from probe dynamics, the TUSO on the Hera probe should be mounted as close to the probe center of gravity as reasonably possible. Although the Hera and CRSC USO's will be contained within thermal ovens, the TUSO and RUSO temperatures should be monitored 
to $\pm 1.0 \mathrm{~K}$. A carefully considered warmup plan is necessary to assure adequate TUSO and RUSO frequency stability. Different USO types (e.g., crystal/Galileo probe and Rubidium/Huygens probe) require significantly different warmup times and steady state power requirements $[1,2,3]$. The Hera probe link frequency and signal strength should be sampled by the CRSC receivers. Upon deployment of the parachute at the top of the atmosphere, the Hera descent latitude/longitude should be reconstructed to $\pm 0.1 \mathrm{deg}$, and the Hera descent speed based on ASI measurements of pressure and temperature should be known to $\pm 0.1 \mathrm{~m} / \mathrm{s}$ [3].

\subsubsection{Specific Calibration Needs}

The short-term frequency stability and long term aging of the TUSO and RUSO should be measured prior to launch. In particular, the repeatability of the frequency drift profile over periods of 30 minutes to several hours should be carefully characterized. After launch, both the TUSO and RUSO should be powered on at least several times during cruise for calibration, and aging and drift characterization.

\subsubsection{TRL and Relevant Heritage}

There are two types of USO technologies with outer solar system entry probe heritage. The Galileo probe and orbiter carried SC-cut quartz crystal USOs, and the Huygens (Titan) probe and Cassini orbiter carried Rb USOs. The Galileo quartz crystal USOs provides a very stable frequency reference for short time periods, although the absolute frequency may be unknown to 100's of Hz, the changes in frequency were the observable of interest. The similarities between the Galileo Jupiter and Hera Saturn probe missions suggests that the crystal USO may be better for the planned Doppler Wind investigation, although further evaluation is needed $[1,2,3,11]$.

\subsubsection{Critical Issues: None}

\subsection{Hera Carrier Camera}

\subsubsection{Measurement Objectives}

The Hera carrier spacecraft will feature a simple visible camera, designed by the Laboratoire d'Astrophysique de Marseille with hardware contributions from the UK and Spain. Design heritage is based on the Rosetta/OSIRIS camera [25]. The purpose of this camera is fourfold:

- To provide optical navigation; 
- To provide contextual imaging of the probe entry site, characterizing the morphology of nearby discrete cloud and haze features, waves and determine local cloud motions with a precision of $3 \mathrm{~m} / \mathrm{s}$;

- To provide a global characterization of Saturn's atmosphere over multiple days during the approach of the carrier;

- To provide contextual imaging of the Saturn system during the approach and departure phase, imaging the planet, rings and diverse satellites.

The camera will accomplish these three requirements with eight carefully selected filters mounted on a filter wheel (see Table 7). In addition, the camera will be also used during the cruise to measure Saturn's brightness continuously and determine the planet's acoustic oscillation modes.

\subsubsection{Design Description}

The design of the camera is dictated by the compromise between the release distance of the probe from Saturn and the angular size of the planet at this distance. A good balance is found with a focal length of $160 \mathrm{~mm}$, which, associated, with the pixel size of the selected detector leads to a resolution of $8.75 \mathrm{arcsec} / \mathrm{pixel}$, when the field of view is close to $3.7 \times 5.0$ square degrees. This compromise will be further studied during Phase A. The optical design is based on a dioptric design using radiation hard glasses, with a pupil diameter of $40 \mathrm{~mm}$, (f/4), allowing exposure times in the 0.1-10 s range in spectral ranges selected by a 8 position filter wheel. The CMOS active pixel sensor CIS115 from e2v (see Fig. 8) is preferred to a CCD mainly for its better radiation hardness and for its smaller pixel size, valuable characteristics for the compactness of the design. Moreover, this detector is pre-qualified for another ESA space mission-JUICE [42]. Its size of $1504 \times 2000$ pixels provides the aforementioned field of view. The detector will be passively cooled to around $-40^{\circ} \mathrm{C}$ to suppress radiation-induced bright pixels. The main structure of the camera is based on a tube made from aluminum alloy or from a similar thermally stable material, depending on the expected environmental conditions of the camera. The camera structure also supports the filter wheel mechanism, and the focal plane assembly, trimmed in position by use of adjustable shims. Three bipods support this structure and limit the possible mechanical stress coming from the bench, on which the instrument is mounted. The electronics box includes the control and command of the detector and of the filter wheel and possibly an automatic control of the exposure time to optimize the dynamics of the current image by analysis of the previous. Compression of the images, in real time, with adjustable compression rates including lossless compressions could also be added, depending on the possible data transmission volume. Packetized data are transmitted to the mass memory of the spacecraft in real time, as the instrument does not include a significant memory. Communications with the spacecraft are based on 2 redundant space wire links, for commands, image data and housekeeping. Without inner memory and without image compression, the image cadence is limited by the rate of this link (1 image per $2.2 \mathrm{~s}$ at a rate of $100 \mathrm{Mbits} / \mathrm{s})$. Dual redundant DC-DC converters supply the various 
sub-systems with appropriate voltages. The electronics is based on the use of one processor (and a second in cold redundancy) ensuring all this task.

\subsubsection{Volume, Mass, Power, Data Rate}

- The masses of the camera and the electronics box are $\sim 4.0$ and $3.5 \mathrm{~kg}$, respectively;

- Length (along the optical axis) of $\sim 200 \mathrm{~mm}$ including the focal plane assembly, without baffle which could add $\sim 100 \mathrm{~mm}$; a transverse axis perpendicular to the fixation plane: $\sim 140 \mathrm{~mm}$; and a transverse axis in the fixation plane: $\sim 120 \mathrm{~mm}$;

- The mean needed power is $\sim 12.0$ watts without image compression, and excluding peaks of power during filter wheel rotations;

- An uncompressed full image represents 6.8 Mbytes, including packetisation and overhead. This can be reduced significantly with different compression strategies.

\subsubsection{Specific/Critical Interface Requirements}

No critical interfaces with the spacecraft exist. Thermal interfaces are still not known but will concern the detector which must be cooled down to a temperature of approximately $-40^{\circ} \mathrm{C}$ to reduce the radiation-induced dark current spikes. This can be done thanks to a passive radiator in direction of a transverse axis of the camera or in direction of its backside, which we assume will be a spacecraft resource. Also, the electronic box has to dump its power mostly by conduction with a spacecraft panel. The only constraint in the spacecraft layout is to have the electronic box close to the focal plane assembly to limit the length of cables and therefore the electromagnetic noise during the readout of the detector.

\subsubsection{Specific Calibration Needs}

The geometric distortion of the optics must be characterized using a star field during flight. A pre-calibration on ground is foreseen, but will not have the accuracy of a calibration in flight.

\subsubsection{TRL and Relevant Heritage}

Given the background of the teams involved in the experiment, only two years of development are needed to reach TRL 6-7. Tables 8 summarizes the current TRL of the camera's elements. 


\section{Proposed mission configuration and profile}

\subsection{Science Mission Profile}

The Saturn probe science mission is a relatively short phase at the end of a relatively long transfer from Earth to Saturn (but shorter than Rosetta's transfer). To provide context for the science mission this section first gives an ordered timeline of all the flight phases of the mission, followed by more detailed discussions of the phases central to delivering the science results.

\subsubsection{End-To-End Mission Profile}

The Saturn probe mission begins its flight phases with a Soyuz-Fregat launch from Kourou on a transfer trajectory to Saturn. Though a thorough search for trajectory options has not yet occurred (planned for the assessment phase), multiple options are expected in the M4 launch window. For study purposes the Hera team has used an example trajectory launching in May 2025, with Venus and Earth gravity assists in August 2025 and July 2026, respectively, arriving at Saturn in August 2033. Variants to this trajectory exist that shorten the trip time by as much as a year, at the expense of larger $\Delta \mathrm{V}$ requirements. No science data acquisition is planned until Saturn approach, so operations for the great majority of the transfer are limited to activities such as trajectory and spacecraft systems monitoring and maintenance. During this period, a system of solar panels and secondary batteries provides electric power. Several weeks priort to Saturn arrival, the CRSC will turn to the proper probe release attitude and will release the Hera probe onto the probe's delivery trajectory, spinning for attitude stability. The probe continues on a ballistic trajectory until entry into Saturn's atmosphere. After probe release the CRSC performs more navigation observations and then a divert maneuver, placing it on a trajectory that allows the CRSC to be properly positioned for the probe data relay. The timing of the probe's release is a trade to be performed in the assessment phase.

The probe entry and descent sequence begins a few hours before entry when the probe event timer begins the "wake up" process, warming the probe's instruments and support systems in preparation for data acquisition and return. Upon encountering the atmosphere, an aerodynamically stable aeroshell enclosing the probe's descent module (DM) will protect the DM from the extreme heat and dynamic forces of entry into Saturn's hydrogen-helium atmosphere at speeds between $26-30 \mathrm{~km} / \mathrm{s}$. By this time the CRSC has begun its $~ 90$-minute overflight of the entry site, aiming the high gain antenna to receive data transmitted from the probe. After the hypersonic deceleration phase is over, the probe's aeroshell is jettisoned, a main parachute opens, slowing the DM's descent in the atmosphere's upper, less dense regions, and the in situ science instruments begin acquiring their data. As the DM descends into denser atmosphere, at an altitude and via a method to be determined in future trade studies, the descent rate will be increased, allowing the DM to reach the required 
depth (plus margin) during the CRSC overflight window. The DM transmits science data to the CRSC for as long as the probe-CRSC relay link survives but to at least the 10 bar pressure level and likely to the 20-bar design margin level or deeper. Eventually the combination of increasing pressures and temperatures will cause the DM systems to fail, then to melt, and finally to vaporize as the DM becomes a new part of Saturn's atmosphere. During the 70-90 minute DM descent the overflying CRSC, operating now on power from primary batteries, maintains the data relay link with the DM, storing multiple copies of the probe's data in redundant onboard storage media for later downlink to Earth. After the data reception window ends the CRSC turns its HGA to Earth and begins the downlink, sending each copy multiple times. The CRSC's Saturn flyby places it on a solar system escape trajectory for spacecraft disposal.

\subsubsection{Core Science Mission Profile}

The Saturn probe's primary science mission closely resembles that of the Galileo probe, and has many similarities to ESA's Huygens probe that successfully entered and descended through Titan's atmosphere. After the warm-up period, the probe begins acquiring science data when its accelerometers detect non-zero acceleration due to atmospheric drag. Until the aeroshell is jettisoned there is no data relay to the CRSC, so the time-tagged accelerometer data and possibly heat shield recession data, needed to reconstruct the vertical profile of atmospheric density, are stored in onboard memory on the DM. When the aeroshell is jettisoned the radio system begins transmitting data from the now-operating in situ instruments, along with the stored data from the entry and deceleration phase. There is no radio receiver on the DM, so there is no real-time commanding capability of the DM after release from the CRSC. The CRSC uses the DM's radio signal, whose carrier frequency is controlled by an onboard ultrastable oscillator (USO), to make Doppler Wind Experiment measurements during the descent, providing a measure of the vertical profile of zonal winds at the descent location. Using a command sequence loaded before release from the CRSC a simple controller on the DM runs a pre-programmed series of measurements by each instrument and routes the data for storage and transmission. The controller uses temperature and pressure data from the Atmospheric Structure Instrument (ASI) to guide instrument modes and observation timing, optimizing the data set for science objectives appropriate to the different atmospheric depths. When the DM reaches the 10-bar level in Saturn's atmosphere, the data return strategy has all probe science data successfully transmitted to the CRSC, satisfying mission success criteria. Subsequent data are returned as the pre-determined (and diminishing) relay data rate allows, according to the controller's priority protocol, until increasing temperatures and pressures cause the DM systems to fail.

Initial analyses indicate that with some Saturn approach trajectories, such as the 2025 EVES trajectory mentioned above, the probe's entry location will be on the sunlit and Earth-facing side of Saturn, providing 90 minutes or more of descent before crossing the evening terminator. This is very beneficial for two potential ex- 
periments. Sunlight intensity measurements by a visible-wavelength channel on the Net Flux Radiometer allow inferring the depth at which solar energy is deposited in Saturn's atmosphere, important in determining what drives Saturn's winds and the overall energy balance of the atmosphere. Receiving the DM's carrier frequency at Earth, possible only when the DM is on the Earth-facing side of Saturn, allows a second Doppler tracking measurement to be made at Earth. This second wind vector component will help separate the line-of-sight wind speed at the probe location into zonal, meridional, and vertical wind speeds.

\subsubsection{Saturn Atmospheric Entry}

Entry into Saturn's atmosphere from hyperbolic approach is a difficult but manageable task. The proposed mission is similar to the Galileo Probe mission that entered Jupiter's atmosphere successfully, deployed the descent probe and collected and transmitted a wealth of data. The Galileo probe entered Jupiter's hydrogen-helium atmosphere at $47.4 \mathrm{~km} / \mathrm{s}$, compared to the $26-30 \mathrm{~km} / \mathrm{s}$ range of entry speeds for the Saturn probe mentioned in Sec. 4.1.1, resulting in a Saturn entry that is significantly less challenging than that faced by Galileo at Jupiter. Figure 9 shows the concept of operation for Galileo entry, deployment, and descent. The Saturn probe's entry and deceleration phase is very similar in most aspects to that of the Galileo mission. A probe scaled from Galileo's $1.27 \mathrm{~m}$ diameter to $1.0 \mathrm{~m}$, with an estimated entry mass of $200 \mathrm{~kg}$ as compared to Galileo's $339 \mathrm{~kg}$, can accomplish the required science at Saturn. Table 9 uses the Galileo equipment as a basis for subsystem masses for the Saturn probe, and indicates that an entry mass of $200 \mathrm{~kg}$ is readily achievable. More rigorous analysis should allow significant reductions in structure mass, since inertial load levels will be much lower than Galileo's design deceleration load of 350 $g$. Although the entry heating rate for a prograde Saturn entry is much less severe than Galileo experienced at Jupiter, Saturn's larger atmospheric scale height yields a long-duration entry resulting in a total heat load that is similar to the Galileo Jupiter entry.

Ablative materials suitable for extreme entry missions and test facilities to qualify Thermal Protection System (TPS) for extreme environments are not yet available to ESA. Since the heritage Carbon Phenolic (HCP) used for the Galileo and PioneerVenus missions is no longer available, NASA's innovative Heatshield for Extreme Entry Environment Technology (HEEET) now under development at NASA's Ames Research Center (ARC) provides a very efficient solution for such an entry profile, resulting in a TPS mass that is only a fraction of the Galileo entry system's TPS mass. NASA plans for this technology to be available at TRL 6 by 2017 for mission teams currently proposing to its 2014 Discovery Program AO. In this context, HEEET is an appropriate technology for a Saturn probe mission within the ESA M4 call schedule and satisfies the maturity requirements stated in the call. The partnership in which NASA would be responsible for the entire entry aeroshell system maximizes the value of ESA's investment in the Saturn probe mission. The proposed partnership with NASA and ARC leverages both the Galileo implementation 
experience and ongoing TPS technology development to enable the Saturn probe mission.

Entry velocity and entry flight path angle (EFPA) strongly influence the atmospheric entry challenge. Saturn's large planetary mass results in typical inertial entry speeds of $36 \mathrm{~km} / \mathrm{s}$ or more, but during a prograde entry Saturn's high rotation rate mitigates up to $10 \mathrm{~km} / \mathrm{s}$ of the entry speed, with the maximum benefit from a nearequatorial entry alignment. Steep EFPAs improve targeting accuracy and reduce the heat load but increase peak deceleration load, heating rate, and peak pressure. Mission success can easily be accomplished with an entry latitude below $10^{\circ}$ and EFPA between $-8^{\circ}$ and $-19^{\circ}$. Table 10 summarizes the range of entry conditions and associated TPS mass for relevant combinations of EFPA and latitude. For all cases, the HEEET material is significantly more mass efficient than the HCP used for Galileo. The benefit is most pronounced at the shallower entry angles, which also provides more benign inertial loads. For steeper EFPAs, the ablative TPS mass is further reduced and is only $10 \%$ of the entry mass. In the study that follows, we primarily focus on the EFPA $=-19^{\circ}$ case, corresponding to a probe entry system mass of 200 $\mathrm{kg}$.

Figure 10 shows the stagnation point heat-flux and impact pressure along trajectories that are bounded by $\pm 10^{\circ}$ latitude (including equatorial) with EFPAs between $-8^{\circ}$ and $-19^{\circ}$. Also shown in this figure are the conditions at which HEEET material has been tested in arc-jet and laser heating facilities. HEEET acreage material is very well behaved at these extreme conditions and at shear levels that are far greater than the anticipated Saturn entry conditions. Adoption of HEEET, in partnership with NASA and ARC, will minimize the TPS technology risk for this mission.

\subsubsection{Probe Delivery to the Entry Trajectory}

Since the entry probe carries no propulsive capability, it is on a ballistic trajectory from the moment of release and the CRSC must establish the proper entry probe trajectory and orientation upon release. The probe also has no attitude control capability, so the CRSC must spin the probe to maintain its attitude until entry. After the long cruise from Earth to Saturn approach the first activities in preparation for release are navigation observations, leading to trajectory correction maneuvers (TCMs) to establish the proper Saturn entry trajectory. The CRSC will release the probe at a distance from Saturn that ensures the entry trajectory will be within tolerances, and might image the departing probe to verify release accuracy and decrease the uncertainty in the probe location. Soon after probe release, the CRSC performs a divert maneuver, changing the CRSC trajectory to a Saturn flyby that provides data relay for the entry probe. The precise timing of probe release is a trade involving navigation accuracy, which degrades with increasing distance from Saturn (earlier release and longer probe coast), the mass of batteries needed to keep the probe warm during its post-release coast, and the mass of propellant needed for the CRSC's divert maneuver, which increases with decreasing distance to Saturn (later release and 
shorter probe coast). Assessment phase studies will estimate the optimum timing of those first navigation activities and TCMs, and probe release.

\subsubsection{Data Relay}

The mission data return strategy uses the data relay method. Studies have shown that this approach yields higher data rates with less operations risk than the directto-Earth (DTE) approach and carries other science benefits as well. Similar to the Galileo probe, after deploying from the entry aeroshell, the descent module transmits data over two independent channels (left and right circular polarization at slightly offset frequencies) through a UHF patch low-gain antenna (LGA) on the DM's upper surface. The CRSC trajectory is within the LGA beam from the start of the probe data transmission through the end of the descent module's mission, some 70-90 minutes later. The CRSC points its high-gain antenna (HGA), feeding a UHF receiver, at the probe entry site, receiving both channels of probe data and storing them onboard in multiple redundant copies. Extremely conservative link analyses based on an $8 \mathrm{~W}$ UHF transmitter suggest data rates of at least 500 bps per channel (the Galileo probe data rate was 128 bps per channel). More refined analysis indicates a variable data rate is feasible, with rates potentially greater than $10 \mathrm{kbps}$ for part of the descent. Performance far greater than the Galileo probe performance is enabled largely by two differences from the Galileo mission: Saturn's lack of intense radiation belts and their associated RF synchrotron radiation noise allows using UHF, which is less attenuated by atmospheric ammonia and water; and the distance from the DM to the CRSC during data relay is between 50,000 and 70,000 $\mathrm{km}$, much closer than the $200,000+\mathrm{km}$ range of the Galileo relay.

After receiving all the probe data onboard, the CRSC downlinks the data to Earth via standard ESA communications facilities. The CRSC will turn its HGA to Earth, transmitting multiple copies of each redundant data file at X-band until the CRSC primary battery charge is effectively exhausted. After recharging its secondary batteries, it then repeats those transmissions periodically as the battery charge allows, until ground commands verify the full data set has been successfully received. Any ancillary data, such as context imaging from a CRSC imager, are included in this downlinked data set.

\subsection{System Level Requirements}

\subsubsection{Entry Probe Requirements}

Between release from the CRSC and atmospheric entry there are three primary requirements on the entry probe: 1) maintain orientation for entry; 2) maintain the probe subsystems and instruments within their environmental tolerances to ensure proper operation during entry and descent; and 3 ) provide adequate timing so the 
"wake-up" sequence begins at the proper time. The CRSC orients the probe and spins it upon release for attitude stability, so the first requirement becomes a requirement on the probe's mass properties: its principle inertial axis must co-align with the aeroshell's symmetry axis. Maintaining environmental conditions is primarily keeping the DM warm at more than 9 AU. In the absence of radioisotope heater units, this will likely require primary batteries to power electric heaters. Batteries would also power an event timer of sufficient accuracy that the wake-up sequence is initiated in time to be completed before entry begins, but not so far in advance that it wastes battery power waiting for entry.

Atmospheric entry involves a different set of requirements. There is a new constraint on the probe's mass properties, along with its exterior geometry: the entire entry system (DM + aeroshell) must be aerodynamically stable at hypersonic speeds, and must maintain that stability in the face of ablative mass and geometry changes. The system must accommodate the extreme heating environment and potentially large inertial loads of atmospheric entry at $26-30 \mathrm{~km} / \mathrm{s}$. If the heat shield is instrumented, the heat shield sensor data must be stored onboard until the sensor and entry accelerometry data can be telemetered to the CRSC after DM deployment from the heat shield. After the end of the hypersonic deceleration phase the DM's controller must initiate a sequence of deployments, including drogue parachute mortar firing, backshell release and main parachute deployment, and heat shield release, for transition to the stable descent phase and primary science data acquisition.

When the DM stabilizes under its main parachute its controller must initiate radio transmission of data to the CRSC, and operation of all science instruments. This continues to a depth determined by the controller using ASI data, when the descent rate must be increased to reach the required 10-bar depth before the CRSC's received signal falls below a margined SNR limit. Potential methods include releasing the main parachute and freefalling or opening a smaller parachute (as per Huygens), reefing the main parachute, or other options, all to be studied in the assessment phase. During this descent phase the DM must maintain its systems within their operating environmental ranges while exposed to exterior temperatures and pressures from $\sim 85 \mathrm{~K}$ at 0.1 bar (near the tropopause) to $\sim 300 \mathrm{~K}$ at 10 bars, possibly increasing to $\sim 350 \mathrm{~K}$ at 20 bars during an "extended mission".

Despite its fundamental nature and extreme importance to planetary science the data volume for the Galileo Probe mission was quite small, less than $1 \mathrm{Mbit}$. The threshold Saturn probe mission data volume will be of similar size. Studies suggest that data rates for the DM-to-CRSC link might support data volumes as high as several tens of Mbit, providing capacity for ancillary science investigations while retaining large margins. Because data acquisition modes change with depth, the data rates from DM instruments are expected to vary during the descent, so some data will need to be stored on the DM prior to transmission. The size of onboard memory required will be studied in the assessment phase, but certainly will be less than the size of the entire data set, and devices with tens of Mbit capacity are small and require little power. 


\subsubsection{Carrier-Relay Spacecraft Requirements}

The Saturn probe mission's CRSC is a fully capable spacecraft that supports the entry probe's mission with a wide variety of functions during cruise, Saturn approach, and the science mission. Preliminary studies resulted in the CRSC requirements discussed here, and produced a system-level spacecraft design and mass budget estimates for assessing feasibility, discussed in Sec. 4.4.

During the Earth-to-Saturn cruise the CRSC provides all functions for delivering the combined spacecraft (probe + CRSC) to Saturn approach, and for maintaining the proper function of its own and the probe's systems, including environmental control, power, and data communications to and from the probe for periodic checkouts and post-launch entry sequence loads. Except for brief periods for activities such as TCMs, the solar-powered CRSC must point its solar arrays sunward, with relatively loose pointing requirements. Communications while in the inner solar system must accommodate uplink for commanding, navigation, and downlink of engineering data over a fairly wide range of Earth-spacecraft-sun angles. Cruise at heliocentric distances greater than $5 \mathrm{AU}$ places more emphasis on power generation and communications. Normal communications will use a $1.5 \mathrm{~m} \mathrm{HGA}$, whose $\mathrm{X}$-band beam width of $\sim 0.75^{\circ} \mathrm{HWHM}$ (Half-width half maximum) sets the spacecraft's pointing accuracy requirement of $0.25^{\circ}$.

Upon Saturn approach the CRSC delivers the probe to its entry trajectory at the proper attitude, and then diverts to a trajectory that allows it to provide data relay support. Required accuracies for navigation, trajectory control, and release attitude control will be studied in the assessment phase, but will not strain current technologies. During the probe's descent the CRSC must continually point its HGA to the entry/descent location to receive the probe's UHF data relay signal. At UHF frequencies the HGA beam width is wide, $\sim 16^{\circ} \mathrm{HWHM}$. Multiple copies of the entire data set must be stored in the CRSC before it downlinks the data to Earth, but those requirements are also easily met with a few hundred Mbit of storage capacity. After the data reception period is over, the CRSC must repoint the HGA to Earth, switch back to X-band, and downlink the data to ground stations. The CRSC must be capable of downlinking each copy of the data set to Earth ground stations at least twice to ensure transfer of the entire set.

In all post-launch phases the CRSC handles all propulsive maneuvers. The postrelease divert maneuver, and a deep space maneuver before the Earth flyby, are the mission's only deterministic maneuvers. The size of the divert maneuver depends upon its timing: the farther from Saturn, the lower the $\Delta \mathrm{V}$ required. At $\sim 30$ days before probe entry that $\Delta \mathrm{V}$ is $\sim 50 \mathrm{~m} / \mathrm{s}(80 \mathrm{~m} / \mathrm{s}$ budgeted); 15 days out it is nearly $100 \mathrm{~m} / \mathrm{s}$. Conservative estimates of statistical $\Delta \mathrm{V}$ budgets and margins indicate 315 $\mathrm{m} / \mathrm{s}$ of $\Delta \mathrm{V}$ capability is sufficient. 


\subsubsection{Ground System Requirements}

The Saturn probe mission uses only standard ground system facilities and resources. The operations team will need standard office, computing, and communications facilities, and access to a mission control facility. Spacecraft commanding and engineering and science data downlink will use standard deep space communications facilities operating at X-band. High-activity periods will include launch, planetary gravity assist flybys, preparations for and execution of probe release and CRSC divert, and the science mission and subsequent data downlink.

\subsection{Launch and Transfer Trajectory}

One of the Saturn probe mission's greatest challenges is sending the spacecraft from Earth to Saturn. Although a thorough study of Earth-to-Saturn trajectories in the M4 time frame has not been done, studies to date suggest there are multiple opportunities and trajectory types to consider. A wider search for Earth-to-Saturn trajectories will be an assessment phase task. The proposal team has used an example trajectory, identified as "EVES 2025" launching in May 2025 with gravity assists at Venus in August of 2025 and Earth in October of 2026, reaching Saturn in October of 2033 (see Figure 11). Launch is to a departure $V_{\infty}$ of $4 \mathrm{~km} / \mathrm{s}\left(\mathrm{C} 3 \mathrm{of} 16 \mathrm{~km}^{2} / \mathrm{s}^{2}\right)$, sufficient to reach Venus, at a declination of $2.76^{\circ}$. The Soyuz-Fregat launch vehicle has a capacity of $1400 \mathrm{~kg}$ to this $V_{\infty}$. There is a deep space maneuver (DSM) between the Venus and Earth flybys with a $\Delta \mathrm{V}$ of $190 \mathrm{~m} / \mathrm{s}$. Statistical $\Delta \mathrm{V}$ includes a launch residuals cancellation maneuver soon after launch, budgeted at $10 \mathrm{~m} / \mathrm{s}$, and trajectory correction maneuvers (TCMs), budgeted at $20 \mathrm{~m} / \mathrm{s}$. Saturn arrival is a few months after the spacecraft's aphelion, at a $V_{\infty}$ of $6.203 \mathrm{~km} / \mathrm{s}$ and approach declination of only $0.077^{\circ}$ (i.e., a nearly equatorial approach). This approach yields a probe entry location well into the sunlit and Earth-facing side of Saturn. Entry at a near-equatorial latitude and at a $-14^{\circ}$ entry flight path angle (EFPA) would be at an atmosphere-relative speed of slightly more than $26 \mathrm{~km} / \mathrm{s}$, near the theoretical minimum for a hyperbolic approach from an Earth-to-Saturn transfer. Entries at higher latitudes, and at steeper EFPAs, would increase that speed somewhat, as discussed in Sec. 4.1.3 above. 30 days before the fly-by, the spacecraft is awakened from hibernation, the CRSC trajectory is adjusted for probe entry targeting and the probe is released. An avoidance manoeuvre (divert manoeuvre) is then performed to provide for the adequate fly-by altitude. The spacecraft then goes back to hibernation to save primary batteries mass and is awakened just prior to the probe mission so the CRSC can support the probe communication relay. 


\subsection{Flight System}

\subsubsection{Entry Probe}

The entry probe element consists of two major sub-elements: the DM that carries all the science instruments and support equipment; and the aeroshell that protects the DM during transfer cruise, post-release cruise, and atmospheric entry, keeping the DM safe from pre-launch until the hypersonic deceleration phase is finished. A 200 $\mathrm{kg}$ probe mass estimate is based on dimensional scaling laws applied to the Galileo probe and first-order adjustments for different instruments and use of the HEEET TPS materials discussed in Sec. 4.1.3 above. No adjustments have been made for the Saturn probe's more benign entry conditions, e.g. lower inertial load, so more detailed study during the assessment phase might realize further mass savings.

\section{Descent Module}

The Descent Module has four primary functions:

- House, control, provide power to, and maintain the operating environment of, science instruments and DM subsystems;

- Collect, store (as needed), and transmit to the CRSC all science and engineering data;

- Control the descent rate profile of the DM to satisfy science objectives and operations requirements;

- Initiate the "wake up" sequence at the proper time before atmospheric entry.

The DM must survive the post-release cruise and the atmospheric entry. Surviving cruise is mostly a matter of electric power for small heaters, along with thermal insulation on the aeroshell exterior. The probe's primary battery complement is sized to include that function. Use of European RHUs would significantly decrease that battery size, but they are not used in this preliminary design due to low TRL. Surviving atmospheric entry involves robustness to large inertial loads of tens to possibly $100 \mathrm{~g}$ 's or more. The DM relies on the aeroshell for protection against the intense heating and huge thermal loads of entry.

All functions except descent rate control would use Galileo techniques. Once it deployed its main parachute the Galileo probe did nothing to control its descent rate. Like the Huygens probe, the Saturn probe cannot afford that simplicity because staying on the unmodified main parachute for the entire descent results in an excessively long descent duration making it impossible to reach 10 bars in the time available for the probe data link. During the assessment phase the DM's descent rate profile and several candidate approaches for controlling the DM's descent rate will be examined. The primary battery approach for the power source is retained, but batteries are now available with higher specific energy resulting in potential mass savings. 
Aeroshell

NASA and NASA Ames Research Center will provide the entire aeroshell, although alternatives from within the European community are being explored. It consists of two main segments, a foreshell and a backshell, and has five primary functions:

- Provide an airframe that is aerodynamically stable at hypersonic and supersonic speeds in an $\mathrm{H}_{2}-\mathrm{He}$ atmosphere, and is spin-stable along its symmetry axis;

- Protect the DM from the intense heating and huge thermal loads of entry;

- During hypersonic entry, accommodate the large inertial loads from the DM;

- Provide a stable transition from supersonic to subsonic flight;

- Upon completion of its entry functions, separate from the DM (by command from the DM).

Section 4.1.3 above treats the entry aspects in detail and discusses probe size and mass. The preliminary studies used an estimated foreshell diameter of $1 \mathrm{~m}$ and a total mass of $200 \mathrm{~kg}$. The aeroshell's role in entry heating protection also gives it a role in post-release survival: its thermal insulating properties aid retention of heat in the DM. The shape of the aeroshell is important. The Galileo probe foreshell, a $45^{\circ}$ sphere-cone, provides heritage for stability and ability to handle the thermal environment. The much lighter backshell of the aeroshell must protect from convective heating by hot gas from the foreshell, and from radiative heating from the trailing shock, where the atmospheric gas "blown open" by the probe's passage "slams shut" again. A partial-sphere shape is appropriate for the backshell, with the entry probe's center of mass at the center of the sphere. With that alignment, odd pressure distributions on the backshell resulting from turbulence or atmospheric winds can cause translational movements but not the much more troublesome angular movements (i.e., rotation) that could destabilize the probe. Transition to subsonic flight overlaps with aeroshell deployment. Most entry aeroshell shapes are unstable during the transition from supersonic to subsonic flight and need stability enhancement. Having the backshell deploy a drogue parachute while at a Mach number comfortably above unity can provide the additional stability, a technique the Galileo probe used. After slowing to subsonic speeds the drogue parachute provides sufficient drag force to pull the backshell from the probe, and then to deploy the DM's main parachute. The main parachute's drag is sufficient to pull the DM from the foreshell.

\subsubsection{Carrier-Relay Spacecraft}

Spacecraft traveling to the outer solar system face three main design challenges: power generation, telecommunications ("telecom"), and thermal control. The Saturn probe mission is a flyby mission, so unlike an orbital mission, propulsion is not a strong design driver. Flight system design work for this proposal did not attempt to optimize the design, but instead provide a conservative proof-of-concept design with sufficient margins to accommodate trades and design work in subsequent phases. All trades done to date will be reopened in the assessment phase and some will 
likely continue after the assessment. The result of work to date is a system-level design that meets all the requirements and fits within the M4 resource constraints and schedules. This section first describes the designs for the three most challenging systems, then describes the remaining systems and configuration.

\section{Power Technology and Power Sizing}

With operations at up to $9.5 \mathrm{AU}$ from the sun, power generation technology was the main driving requirement of the preliminary design task. This mission would benefit greatly from nuclear power sources, whether for power generation or for heating (RHUs), but use of European launchers requires use of European nuclear technology for launch pad safety qualification. The cost and low TRL of European space nuclear technologies makes that option difficult to achieve in the M4 time frame, so they were not used in this preliminary study. Fortunately the mission's brief science operations phase requires relatively little energy. A solar power system supplemented by primary and secondary batteries is sufficient:

- The need for survival heating at up to $9.5 \mathrm{AU}$ and for an on-board clock sets the solar array size. A low-activity power demand of $140 \mathrm{~W}$ requires a solar array output of $170 \mathrm{~W}$ at $9.5 \mathrm{AU}$, provided by a classical rigid solar array with LILT cells, with a surface area of about $53 \mathrm{~m}^{2}$;

- Secondary batteries provide energy for safe mode during cruise and allow for temporary off-sun pointing before arrival at Saturn (for TCMs, probe spinup and release, etc.);

- Primary batteries supplement the system during the science operations phase. A supplemental capacity of $16.6 \mathrm{kWh}$ meets the $400 \mathrm{~W}$ power demand during science operations (with telecom system transmission) for a $64 \mathrm{~h}$ time period.

For conservatism in this preliminary study we used the highest-TRL solutions for the solar array and primary batteries:

- Standard rigid solar array using Spectrolab LILT cells (currently flying on Juno);

- Saft LSH20 primary batteries currently flying on the Rosetta mission's Philae lander.

A delta-qualification will be required for the LILT cells (qualified in Jupiter conditions) to qualify them for Saturn conditions. In the current baseline, the Solar Array is provided by the US. To further expand qualification status to Saturn, Spectrolab's heritage XTJ (29.2\% eff) solar cells can be screened and tested for operation under 9.5 AU LILT conditions using their X-25 solar simulator with specially tuned neutral density filters. Based on their experience on Juno, Spectrolab's assessment is that the probability of success is elevated and that the TRL of 5 can be acquired in parallel with the Assessment Phase before 2018.

The Solar Array and the primary batteries would however represent then more than one third of the CRSC dry mass. During the assessment phase, lower TRL 
options can be traded so as to decrease the mass and/or provide more operational time, including:

- Flexible solar array design;

- Concentrator-based solar array design (see Spectrolab's suggestions in Annex);

- More performing primary batteries;

- Decrease hibernation/survival heating need thanks to RHUs.

\section{Telecommunications and Data Handling}

For communications from the CRSC to Earth the telecom system uses a $65 \mathrm{~W}$ Xband TWTA through a $1.5 \mathrm{~m}$ 2-axis steerable HGA, providing 4-5 kbps downlink at 9.5 AU. LGAs ensure communication with Earth when the spacecraft is in Earth's vicinity, and low-rate communications in safe mode. Data reception from the probe also uses the HGA, feeding a UHF receiver. The mission's data volume is quite small, possibly several tens of Mbit or less, so depending on the choice of on-board computer, the computer's standard memory could store multiple copies of the probe data. If necessary, a small supplemental memory device can expand available memory to meet the storage requirement. Each copy of the probe data is downlinked multiple times for redundancy.

\section{Thermal}

Given the large range of heliocentric distances for any Earth-to-Saturn trajectory, maintaining an acceptable thermal environment for spacecraft subsystems without excessive power requirements is a non-trivial task. Particular attention must be devoted to propulsion system propellant tanks and feed lines to externally mounted thrusters. Power needed to keep those components warm when more than $5 \mathrm{AU}$ from the sun is a significant part of the solar array sizing budget. The Rosetta mission successfully addressed slightly less demanding but nonetheless similar challenges. With careful attention during design to heat loss pathways and appropriate use of lightweight insulating materials, high-TRL materials and techniques will suffice for a robust thermal management system.

\section{Propulsive Delta-V}

To reduce mass a MON-MMH bi-propellant subsystem is adopted for this preliminary study. No main engine is required: $10 \mathrm{~N}$ thrusters are sufficient. Propellant temperature must be considered since propulsion system heating to prevent propellant freezing is a major factor in the survival-heating budget, which sizes the solar array.

Table 11 shows the $\Delta \mathrm{V}$ and propellant budget for the trajectory presented in Sec. 4.3 , assuming a dry mass of $1115 \mathrm{~kg}$ including the $20 \%$ system margin $(875 \mathrm{~kg}$ dry 
after probe release), a propellant need of $9 \mathrm{~kg}$ for GNC, times two to account for the $100 \%$ required margin, and $2 \%$ of residuals.

\section{Pointing Modes and AOCS Design}

The CRSC is designed to remain sun-pointed throughout cruise except for brief periods for activities such as TCMs and probe release. For any trajectory that stays beyond $0.7 \mathrm{AU}$ the LILT solar cells are compatible with full sun exposure at Venus and do not require off-pointing when close to the Sun. Safe mode is also sun-pointed. The cruise mode is in slow spin, interrupted only for TCMs and Earth communications, which use the HGA's two-axis pointing mechanism so the solar arrays stay sun-pointed. The mission uses a hibernation mode similar to that of the Rosetta mission. Beyond about 5.5 AU, the solar array output is insufficient to power continuous operation, requiring hibernation from $5.5 \mathrm{AU}$ to Saturn approach. It can be used also inside of 5.5 AU to decrease operations costs. During hibernation the CRSC is sun-pointed, in slow spin. The solar array panel arrangement aids stability by establishing the maximum moment of inertia around the spin axis.

At the proper time before the fly-by (to be established in the assessment phase), the CRSC will wake up, send status data to Earth to verify proper spacecraft function, and perform navigation tasks to allow design and execution of a TCM, if needed. At the proper time it enters the inertial pointed mode, executes the TCM, turns to the attitude needed for probe release, releases the probe, turns to the divert maneuver attitude, performs the maneuver, and then returns to sun-pointed hibernation mode. Studies during the assessment phase will establish the optimum timing for this sequence.

For the data relay fly-by the CRSC will be sun-pointed in inertial pointing mode. It will wake up, point its HGA towards the Earth for a systems check, then towards the probe entry site for receiving the probe's data while it descends, then towards the Earth again for data downlink. If reaction wheels are used, duty cycles will be very limited and will allow use of very light wheels. Without reaction wheels, $1 \mathrm{~N}$ fine hrusters may be used instead. This tradeoff will be investigated during the assessment phase.

\subsubsection{Probe Delivery}

The time between probe release and entry will be investigated to optimize probe primary batteries mass and propellant mass required for the post-release divert maneuver. The shorter this time, the lighter the probe batteries, but the higher the required $\Delta \mathrm{V}$ for the CRSC divert maneuver. For these preliminary studies we assumed release 30 days before entry. Navigation and attitude control accuracy requirements will be investigated as well. 


\subsubsection{Mass Budget}

Table 12 presents nominal mass estimates based on similar subsystems from previous missions. The values are given in maximum estimated mass (best estimate plus maturity margin) so they can be compared to similarly-sized past planetary missions. A 20\% system margin is added for a conservative estimate of propellant mass and wet mass. Adding the launch adapter (Soyuz 937mm diameter interface) yields the mass to be compared with the mass capacity from the trajectory analysis. The dry mass structural index used is comparable to same-sized planetary missions: this is conservative as the CRSC will have fewer components than an orbiter. The resulting mass is compatible with the Soyuz launch scenario presented in Section 4.3.

\subsubsection{Configuration}

A preliminary configuration design allowed checking for fundamental physical architecture issues. One suitable face allocation was found but many other combinations may prove of interest during future phases. We show here an example of a 6-faced physical architecture including:

- The launcher interface face, also hosting the main thrusters; this face is sunpointed in cruise;

- Two lateral faces each supporting one solar array wing $\left(27 \mathrm{~m}^{2}\right.$ each);

- One lateral face supporting the HGA during launch;

- One lateral face for CRSC bus units;

- One top face bearing the probe.

The solar array is deployed immediately after launch so that its cells are towards the launcher interface face. The HGA is then deployed towards this face. Figure 12 shows a 7-panel solar array arrangement stowed and deployed. This 1-3-3 arrangement has flown on telecommunication satellites like Ciel2 with bigger panels. It yields a large moment of inertia about the spin axis for robust spin stability.

\section{Management scheme}

\subsection{Management Overview}

The Hera mission for an in situ atmospheric probe of Saturn is proposed as an ESA-led mission, with a significant and essential contribution by NASA. The Proposal consortium is led by Dr. Olivier Mousis of France and Dr. David Atkinson of the USA. Dr. Mousis is Professor of Astrophysics at Aix-Marseille University and Member of the Institut Universitaire de France, and Dr. David H. Atkinson, a 
Professor of Electrical and Computer Engineering at the University of Idaho and a JPL Research Affiliate. Dr. Atkinson has participated in several Saturn Probe mission concept studies, with a particular focus on Saturn probes under the NASA New Frontiers program. Participating in the Proposal Consortium are two industrial companies, Thales Alenia Space France and The Boeing Company in Huntington Beach, California, USA. Additionally, six science teams will participate in the Phase A Study, representing six potential science instruments for the Hera mission. The industrial partner participation is outlined in Subsection 5.2 of the Management Section, and the Science Teams are outlined in Subsection 5.4, Instrument Consortia.

If Hera is selected for flight following Phase A then a joint ESA-NASA mission management will be established under the responsibilities of both agencies. ESA and NASA will follow their own approach for the industrial activities. At the appropriate time during the study phase, ESA will select its industrial contractor for the study phase B1, (or contractors if parallel competitive studies are being conducted) and in a second step the Hera development industrial contractor (Phase B/C/D/E/F).

\subsection{International Collaboration}

The international collaboration for the Hera mission will involve the Spacecraft carrier bus and atmospheric probe as well as the science instruments and science investigations. The Phase A study of spacecraft structures and systems will involve selected industrial partners, as well as NASA Ames Research Center and Jet Propulsion Laboratory. The selected European industrial partner will study the architecture for the Carrier Spacecraft Bus and the Probe. The selected American industrial partner will contribute by studying the solar power system, including solar arrays and the power management and distribution system. NASA Ames Research Center will contribute by studying the probe entry system, including the Thermal Protection System (TPS) for foreshell and backshell and the associated underlying structure including aero-thermodynamic.

The Hera instrument payload will be provided by instrument PI teams from ESA's Members states and NASA scientific communities. Payload funding for ESA's members states will be provided by National funding agencies. US payload contribution will be funded by NASA. The lead-funding agency for each PI-team will either be the PI National Funding Agency for a European PI-led team and NASA for a US-led PI team. NASA funding decisions will not be made prior to selection for the Phase A study.

\subsection{Procurement}

ESA and selected industrial partners will lead the Phase A study for major spacecraft systems procurement. Two major subsystems are anticipated to be supplied 
by the United States, either by direct procurement by ESA or by contribution from NASA (according to negotiations for a Memorandum of Understanding between ESA and NASA). These two major subsystems are the solar electric power system (including solar arrays and the power management and distribution system), and the probe entry system (including the TPS material and the heatshield and backshell structures). Services that may be supplied by NASA include trajectory planning and analysis (possibly from the Jet Propulsion Laboratory in Pasadena) and deep space tracking from NASA's Deep Space Network (DSN).

\subsection{Instrument Consortia}

Six science instruments will be studied in Phase A for cost and science benefit. Each of these instrument study teams will be an international collaboration. The different science instrument consortia are described below and summarized in Table 13.

\subsubsection{Science Camera (Carrier)}

The science team will be led by Olivier Mousis (PI) of Laboratoire d'Astrophysique de Marseille (France), with support from Leigh Fletcher (Co-PI) of University of Oxford, Clarendon Laboratory (UK), and Ricardo Hueso, Universidad del País Vasco UPV/EHU, Spain. The different elements of the Science Camera will be provided as follows:

- optics, mechanics and main electronic: Patrick Levacher, Laboratoire d'Astrophysique de Marseille (France), François-Xavier Schmider, Observatoire de la Côte d'Azur (France);

- CMOS chip and proximity electronics: James Endicott, e2v (UK), Andrew Holland, Open University (UK);

- filter wheel will be provided by Christina Ortega, AVS (Spain), Miguel Angel Carrera, AVS (Spain).

\subsubsection{Atmospheric Structure Investigation (Probe)}

The science team will be led by Francesca Ferri of Universit degli Studi di Padova (Italy), with participation of Anthony Colaprete of NASA Ames Research Cener (USA) and Georg Fischer of the Austrian Academy of Sciences, Graz (Austria). Provision of the individual sensors of the ASI will be decided during the Phase A study. 


\subsubsection{Mass Spectrometer (Probe)}

The science team will be led by Peter Wurz of Bern University (PI, Switzerland) and Jack Hunter Waite, SouthWest Research Institute (Co-PI, USA). The different elements of the Mass Spectrometer will be provided as follows:

- mass spectrometer, gas inlet and interface to sub-units, with all its electronics, system responsibility, integration will be provided by Peter Wurz, Bern University/Switzerland;

- the Cryotrap, getter traps, vacuum pumps, complete with valves, gauges, and associated electronics will be supplied by Jack Hunter Waite, Southwest Research Institute, SWRI (USA);

- the Gas calibration system $(\mathrm{H} / \mathrm{He})$, noble gases abundance and isotopes (TBC), other isotopes references (e.g. $\mathrm{C}$ and $\mathrm{H}$ ) will be supplied by Andrew Morse and Simon Sheridan, Open University (UK).

\subsubsection{Radio Science (Probe and Carrier)}

The hardware for the Radio Science investigation consists of the ultra-stable oscillator in the probe Communication system and the receiver on the carrier that is capable of measuring frequency and probe signal AGC. These will be part of the Communication system provided by the European industrial partner selected by ESA in Phase A. The radio science investigation will be led by David Atkinson of the University of Idaho (USA), assisted by Michael Bird (DE) and Thomas Spilker (USA). An additional European investigator may be added to the radio science team during Phase A.

\subsubsection{Nephelometer (Probe)}

The science team will be led by by Daphne Stam, of Delft Space Institute, Technical University Delft (Netherlands), with assistance by Jean-Baptiste Renard (Co-PI) and Gwenael Berthet, LPC2E-CNRS /Orléans University, Olga Munoz, Instituto de Astrofisica de Andalucia, CSIC (Spain), (France), and Don Banfield, Cornell University (USA). The different elements of the Nephelometer will be provided as follows:

- the Light Optical Aerosol Counter (LOAC) will be provided by Jean-Baptiste Renard, LPC2E-CNRS (France);

- the Polarimetric Aerosol Versatile Observatory (PAVO) optics will be provided by Christoph Keller, Leiden University (Netherlands) and Frans Snik (Netherlands);

- PAVO electronics and detector will be provided by Daphne Stam, Technical University Delft (Netherlands). 


\subsubsection{Net Flux Radiometer (Probe)}

The science team will be led by Michael Amato (PI), Shahid Aslam, Conor Nixon, Don Jennings and Tilak Hewagama from NASA Goddard Spaceflight Center (GSFC). The different elements of the Net Flux Radiometer will be provided as follows:

- the Net Flux Radiometer Instrument (detector, electronics, optics, thermal, mechanical) will be provided by Shahid Aslam, Michael Amato, and NASA GSFC engineering and instrument management groups;

- the Detector will be provided by Ernst Kessler of IPHT Jena, Germany, and the rad hard ROIC will be provided by NASA GSFC;

- the filters will be provided by Simon Calcutt of Oxford University, UK.

\subsection{Data Policy}

The main repository for an ESA-led Planetary mission is ESA's Planetary Science Archive (PSA). Science instrument data will be archived in a timely manner in ESA's PSA, and the data will be mirrored to NASA's Planetary Data System (PDS).

Our project plans to share these experiment data with the outer planet community through participation at symposia and workshops. Additionally, we will present papers and posters at relevant planetary science professional meetings and workshops, such as the European Geosciences Union (EGU), the American Geophysical Union (AGU), the European Planetary Science Congress (EPSC), the Lunar and Planetary Science Conference, NASA's Outer Planet Analysis Group (OPAG), and the DPS Unit of American Astronomical Society. A special effort will be made to collaborate with the Exoplanet community in achieving a broader context for the Hera probe findings, for example, with participation in the European Astrobiology Conference. Papers detailing research results will be submitted to professional journals.

\subsection{Education and Public Outreach}

The interest of the public in the Saturnian system continues to be significant, with much of the credit for the high interest in Saturn due to the extraordinary success of the Cassini-Huygens mission. Images from the Saturnian system are regularly featured as the NASA "Astronomy Picture of the Day", and continue to attract the interest of the international media. The interest and excitement of students and the general public can only be amplified by a return to Saturn. The Hera mission will hold appeal for students at all levels. Education and Public Outreach activities will be an important part of the Hera mission planning. An EPO team will be created to develop programs and activities for the general public and students of all ages. Additionally, Hera results and interpretation of the science will be widely distributed to 
the public through internet sites, leaflets, public lectures, TV and radio programmes, CD and DVDs, museum and planetarium exhibitions, and in popular science magazines and in newspapers.

\section{Costing}

Based on the mission elements defined in Sec. 4, a preliminary cost study performed by CNES suggests that the total budget of a Saturn mission consisting of a probe and a carrier is $\sim 493$ million $€$, excluding the Science payload. Table 14 summarizes the total cost for ESA, estimated to be $\sim 414$ million $€$. It includes the Soyuz Launch, the Carrier Relay Spacecraft (CRSC), the Hera probe (descent module), the ground segment, the operations-exploitation and internal ESA costs.

Table 15 lists the cost of elements supplied by NASA, whose total is found about 79 million of euros. The cost of solar arrays has been scaled to that of the JUICE mission and a rough estimate of the budget needed for the thermal protection shield has been provided by NASA Ames.

\section{Summary and conclusion}

In situ exploration of the giant planets is necessary to further constrain models of solar system formation and chemical/thermal evolution, and the origin and evolution of atmospheres, to provide a basis for comparative studies of the gas and ice giants, and to provide a unique groundtruth for studying extrasolar planetary systems. In addition, the gas and ice giants provide a laboratory for which the atmospheric chemistries, dynamics, processes, and interiors of all the planets including Earth can be studied.

Within the deeper, well-mixed atmospheres of the giant planets the most pristine material from the epoch of solar system formation can be found, providing clues to the local chemical and physical conditions existing at the time and location at which the planet formed. In particular, measurements of noble gas abundances are needed to understand the formation and evolution of the giant planets. In addition to the absolute abundance of noble gases, the ratio of the noble gas abundances will strongly constrain how, when, and where the giant planets formed. Perhaps most important, the abundance of helium will indicate the extent to which helium/hydrogen phase separation has occurred in the deep interior of the giant planets. A Saturn entry probe can determine whether the enhancement of heavy noble gases found in Jupiter by Galileo is a general feature of all the giant planets, and measurements of the Saturn helium abundance can be contrasted with the Jupiter helium abundance measured by the Galileo probe, leading to a self-consistent theory for the thermal evolution of both Jupiter and Saturn. This theory will then present a ground truth to calibrate theories of the evolution of giant planet formation, including exoplanets. 
The key goal of a Saturn entry probe mission is to measure the abundances of the noble gases $\mathrm{He}, \mathrm{Ne}, \mathrm{Ar}, \mathrm{Kr}, \mathrm{Xe}$ and their isotopes, the heavier elements $\mathrm{C}, \mathrm{N}$, and $\mathrm{S}$, key isotope ratios ${ }^{14} \mathrm{~N} /{ }^{15} \mathrm{~N},{ }^{12} \mathrm{C} /{ }^{13} \mathrm{C}$ and $\mathrm{D} / \mathrm{H}$, and disequilibrium species such as $\mathrm{CO}, \mathrm{PH}_{3}, \mathrm{AsH}_{3}, \mathrm{GeH}_{4}$ which act as tracers of internal processes, and can be achieved by a Saturn entry probe reaching 10 bars.

Comprising a single probe accompanied by a Carrier Relay Spacecraft, the Hera Saturn atmospheric entry probe has been proposed as an ESA medium class (Mclass) mission. Hera will measure the noble gas, chemical, and isotopic compositions, processes, and dynamics of Saturn's upper atmosphere, providing science essential for elucidating the origin, formation and thermal and chemical evolution of Saturn, the giant planets, and the solar system, and to provide ground truth measurement for extrasolar planet science. Hera will probe far below regions accessible to remote sensing, well into the cloudforming region of the troposphere to locations where the most important cosmogenically abundant species well mixed. Along the probe descent, Hera will provide in situ tracking of Saturn's atmospheric dynamics including zonal winds, waves, convection and turbulence, and measurements of atmospheric pressure and temperature, and the location, density, and composition of the upper cloud layers.

By leading to an improved understanding of the processes by which giant planets formed, including the composition and properties of the local solar nebula at the time and location of giant planet formation, Hera will extend the legacy of the Galileo and Cassini missions by further addressing the creation, formation, and chemical, dynamical, and thermal evolution of the giant planets, the entire solar system including Earth and the other terrestrial planets, and formation of other planetary systems.

Acknowledgements O.M. acknowledges support from CNES. This work has been carried out thanks to the support of the A*MIDEX project ( $n^{\circ}$ ANR-11-IDEX-0001-02) funded by the "Investissements d'Avenir" French Government program, managed by the French National Research Agency (ANR).

\section{References}

1. Asmar, S.W., D.H. Atkinson, G. Weaver, and N. Yu.: Science-Quality Oscillators for Deep Space Probes, 7th International Planetary Probe Workshop, Barcelona, Spain (2010).

2. Asmar, S. W., Atkinson, D. H., Bird, M. K., Wood, G. E. 2004. Ultra-stable oscillators for planetary entry probes. Planetary Probe Atmospheric Entry and Descent Trajectory Analysis and Science 544, 131-134 (2004).

3. Atkinson, D. H., Pollack, J. B., Seiff, A.: The Galileo probe Doppler wind experiment: Measurement of the deep zonal winds on Jupiter. Journal of Geophysical Research 103, 22911-22928 (1998).

4. Atkinson, D. H., Ingersoll, A. P., Seiff, A.: Deep winds on Jupiter as measured by the Galileo probe. Nature 388, 649-650 (1997).

5. Atkinson, D. H., Pollack, J. B., Seiff, A.: Galileo Doppler Measurements of the Deep Zonal Winds at Jupiter. Science 272, 842-843 (1996). 
6. Atkinson, D. H.: Measurement of Planetary Wind Fields by Doppler Monitoring of an Atmospheric Entry Vehicle. Ph.D. Thesis (1989).

7. Atreya, S. K., Wong, M. H., Owen, T. C., Mahaffy, P. R., Niemann, H. B., de Pater, I., Drossart, P., Encrenaz, T.: A comparison of the atmospheres of Jupiter and Saturn: deep atmospheric composition, cloud structure, vertical mixing, and origin. Planetary and Space Science 47, 12431262 (1999).

8. Balsiger, H., and 49 colleagues.: ROSINA - Rosetta Orbiter Spectrometer for Ion and Neutral Analysis. Space Science Reviews 128, 745-801 (2007).

9. Boese, R. W., Twarowski, R. J., Gilland, J., Hassig, R. E., Brown, F. G.: The infrared radiometer on the sounder probe of the Pioneer Venus mission. IEEE Transactions on Geoscience and Remote Sensing 18, 97-100 (1980).

10. Bird, M. K., and 14 colleagues.: The vertical profile of winds on Titan. Nature 438, 800-802 (2005).

11. Bird, M. K., Heyl, M., Allison, M., Asmar, S. W., Atkinson, D. H., Edenhofer, P., Wohlmuth, R., Iess, L., Tyler, G. L., Plettemeier, D.: The Huygens Doppler Wind Experiment. Huygens: Science, Payload and Mission 1177, 139 (1997).

12. Chambers, J. E., Wetherill, G. W.: Planets in the asteroid belt. Meteoritics and Planetary Science 36, 381-399 (2001).

13. Durry, G., Hauchecorne, A., Ovarlez, J., Ovarlez, H., Pouchet, I., Zeninari, V.: In situ Measurement of $\mathrm{H}_{2} \mathrm{O}$ and $\mathrm{CH}_{4}$ with Telecommunication Laser Diodes in the Lower Stratosphere: Dehydration and Indication of a Tropical Air Intrusion at Mid-Latitudes. J. Atm. Chem. 43(3), 175-194 (2002).

14. Fletcher, L. N., Baines, K. H., Momary, T. W., Showman, A. P., Irwin, P. G. J., Orton, G. S., Roos-Serote, M., Merlet, C.: Saturn's tropospheric composition and clouds from Cassini/VIMS 4.6-5.1 $\mu \mathrm{m}$ nightside spectroscopy. Icarus 214, 510-533 (2011).

15. Fletcher, L. N., Orton, G. S., Teanby, N. A., Irwin, P. G. J..: Phosphine on Jupiter and Saturn from Cassini/CIRS. Icarus 202, 543-564 (2009).

16. Folkner, W. M., and 16 colleagues.: Winds on Titan from ground-based tracking of the Huygens probe. Journal of Geophysical Research (Planets) 111, E07S02 (2006).

17. Folkner, W. M., Woo, R., Nandi, S.: Ammonia abundance in Jupiter's atmosphere derived from the attenuation of the Galileo probe's radio signal. Journal of Geophysical Research 103, 22847-22856 (1998).

18. Folkner, W. M., Preston, R. A., Border, J. S., Navarro, J., Wilson, W. E., Oestreich, M.: Earthbased radio tracking of the Galileo probe for Jupiter wind estimation. Science 275, 644-646 (1997).

19. Fulchignoni, M., and 42 colleagues.: In situ measurements of the physical characteristics of Titan's environment. Nature 438, 785-791 (2005).

20. Fulchignoni, M., and 27 colleagues.: The Characterisation of Titan's Atmospheric Physical Properties by the Huygens Atmospheric Structure Instrument (Hasi). Space Science Reviews 104, 397-434 (2002).

21. García-Melendo, E., Pérez-Hoyos, S., Sánchez-Lavega, A., Hueso, R.: Saturn's zonal wind profile in 2004-2009 from Cassini ISS images and its long-term variability. Icarus 215, 62-74 (2011).

22. Gomes, R., Levison, H. F., Tsiganis, K., Morbidelli, A.: Origin of the cataclysmic Late Heavy Bombardment period of the terrestrial planets. Nature 435, 466-469 (2005).

23. Grard, R., Svedhem, H., Brown, V., Falkner, P., Hamelin, M.: An experimental investigation of atmospheric electricity and lightning activity to be performed during the descent of the Huygens Probe onto Titan. Journal of Atmospheric and Terrestrial Physics 57, 575-585 (1995).

24. Hansen, J. E., Travis, L. D.: Light scattering in planetary atmospheres. Space Science Reviews 16, 527-610 (1974).

25. Keller, H. U., and 68 colleagues.: OSIRIS - The Scientific Camera System Onboard Rosetta. Space Science Reviews 128, 433-506 (2007).

26. Mahaffy, P. R., and 84 colleagues.: The Sample Analysis at Mars Investigation and Instrument Suite. Space Science Reviews 170, 401-478 (2012). 
27. Milos, F. S.: Galileo probe heat shield ablation experiment. Journal of Spacecraft and Rockets 34, 705-713 (1997).

28. Mousis, O., and 50 colleagues.: Scientific rationale for Saturn's in situ exploration. Planetary and Space Science 104, 29-47 (2014).

29. Niemann, H. B., and 18 colleagues.: The Gas Chromatograph Mass Spectrometer for the Huygens Probe. Space Science Reviews 104, 551-590 (2002).

30. Niemann, H. B., and 12 colleagues.: The Galileo Probe Mass Spectrometer: Composition of Jupiter's Atmosphere. Science 272, 846-849 (1996).

31. Orton, G. S., and 16 colleagues: Characteristics of the Galileo probe entry site from Earthbased remote sensing observations. Journal of Geophysical Research 103, 22791-22814 (1998).

32. Owen, T., Mahaffy, P., Niemann, H. B., Atreya, S., Donahue, T., Bar-Nun, A., de Pater, I.: A low-temperature origin for the planetesimals that formed Jupiter. Nature 402, 269-270 (1999).

33. Quilligan, G., et al.: A $0.18 \mu \mathrm{m}$ CMOS Thermopile Readout ASIC Immune to $50 \mathrm{Mrad}$ Total Ionizing Dose $(\mathrm{Si})$ and Single Event Latchup to $174 \mathrm{MeV}-\mathrm{cm}^{2} / \mathrm{mg}$. International Workshop on Instrumentation for Planetary Missions (IPM-2014), Greenbelt, MD (2014).

34. Ragent, B., Privette, C. A., Avrin, P., Waring, J. G., Carlston, C. E., Knight, T. C. D., Martin, J. P.: Galileo Probe Nephelometer Experiment. Space Science Reviews 60, 179-201 (1992).

35. Read, P. L., Conrath, B. J., Fletcher, L. N., Gierasch, P. J., Simon-Miller, A. A., Zuchowski, L. C.: Mapping potential vorticity dynamics on saturn: Zonal mean circulation from Cassini and Voyager data. Planetary and Space Science 57, 1682-1698 (2009).

36. Rietjens, J. H. H., van Harten, G., Bekkers, D., et al.: Performance of spectrally modulated polarimetry II: Data reduction and absolute polarization calibration of a prototype SPEX satellite instrument, Appl. Optics, submitted.

37. Sanchez-Lavega, A., Colas, F., Lecacheux, J., Laques, P., Parker, D., Miyazaki, I.: The Great White SPOT and disturbances in Saturn's equatorial atmosphere during 1990. Nature 353, 397401 (1991).

38. Seiff, A., Kirk, D. B., Knight, T. C. D., Young, R. E., Mihalov, J. D., Young, L. A., Milos, F. S., Schubert, G., Blanchard, R. C., Atkinson, D.: Thermal structure of Jupiter's atmosphere near the edge of a $5-\mu \mathrm{m}$ hot spot in the north equatorial belt. Journal of Geophysical Research 103, 22857-22890 (1998).

39. Seiff, A., Knight, T. C. D.: The Galileo Probe Atmosphere Structure Instrument. Space Science Reviews 60, 203-232 (1992).

40. Seiff, A., Kirk, D. B., Young, R. E., Blanchard, R. C., Findlay, J. T., Kelly, G. M., Sommer, S. C.: Measurements of thermal structure and thermal contrasts in the atmosphere of Venus and related dynamical observations - Results from the four Pioneer Venus probes. Journal of Geophysical Research 85, 7903-7933 (1980).

41. Snik, F., Karalidi, T., Keller, C. U.: Spectral modulation for full linear polarimetry. Applied Optics 48, 1337-1346 (2009).

42. Soman, M., Holland, A. D., Stefanov, K. D., Gow, J. P., Leese, M., Pratlong, J., Turner, P.: Design and characterisation of the new CIS115 sensor for JANUS, the high resolution camera on JUICE. Society of Photo-Optical Instrumentation Engineers (SPIE) Conference Series 9154, 915407 (2014).

43. Sromovsky, L. A., Collard, A. D., Fry, P. M., Orton, G. S., Lemmon, M. T., Tomasko, M. G., Freedman, R. S.: Galileo probe measurements of thermal and solar radiation fluxes in the Jovian atmosphere. Journal of Geophysical Research 103, 22929-22977 (1998).

44. von Zahn, U., Hunten, D. M., Lehmacher, G.: Helium in Jupiter's atmosphere: Results from the Galileo probe helium interferometer experiment. Journal of Geophysical Research 103, 22815-22830 (1998).

45. Webster, C. R., Mahaffy, P. R.: Determining the local abundance of Martian methane and its ${ }^{13} \mathrm{C} /{ }^{12} \mathrm{C}$ and $\mathrm{D} / \mathrm{H}$ isotopic ratios for comparison with related gas and soil analysis on the 2011 Mars Science Laboratory (MSL) mission. Planetary and Space Science 59, 271-283 (2011).

46. Wong, M. H., Mahaffy, P. R., Atreya, S. K., Niemann, H. B., Owen, T. C.: Updated Galileo probe mass spectrometer measurements of carbon, oxygen, nitrogen, and sulfur on Jupiter. Icarus 171, 153-170 (2004). 
47. Wurz, P., Abplanalp, D., Tulej, M., Lammer, H.: A neutral gas mass spectrometer for the investigation of lunar volatiles. Planetary and Space Science 74, 264-269 (2012).

48. Zarnecki, J. C., Ferri, F., Hathi, B., Leese, M. R., Ball, A. J., Colombatti, G., Fulchignoni, M.: In-flight performance of the HASI servo accelerometer and implications for results at Titan. ESA-SP-544, 71-76 (2004). 
Table 1 Science Priorities. Priority 1 measurements focus only on questions related to Saturn's origin. Science Priorities 2 and 3 address questions related to the structure of Saturn's atmosphere and its formation conditions.

\section{Priority 1 measurements}

- 1.1 The atmospheric fraction of $\mathrm{He} / \mathrm{H}_{2}$ with $2 \%$ accuracy on the measurement (same accuracy as Galileo). A firm measurement of the He abundance is needed to constrain Saturn's interior;

- 1.2 The abundances on the chemically inert noble gases $\mathrm{Ne}, \mathrm{Xe}, \mathrm{Kr}$ and $\mathrm{Ar}$ with $10 \%$ accuracy on the measurement (uncertainties close to those in solar abundances). These elements constitute excellent tracers for the materials in the subreservoirs existing in the PSN;

- 1.3 The vertical profiles of elemental enrichments in cosmogenically abundant species $\mathrm{C}$, $\mathrm{N}$ and $\mathrm{S}$. $\mathrm{C} / \mathrm{H}, \mathrm{N} / \mathrm{H}$ and $\mathrm{S} / \mathrm{H}$ should be sampled with accuracies better than $\pm 5 \%$ (same accuracy as Galileo). The precise measurement of these species provides clues regarding the disk's thermodynamic conditions at the epoch of Saturn's formation.

Priority 2 measurements

- 2.1 The isotopic ratios in hydrogen $(\mathrm{D} / \mathrm{H})$, helium ${ }^{3} \mathrm{He} /{ }^{4} \mathrm{He}$, carbon $\left({ }^{12} \mathrm{C} /{ }^{13} \mathrm{C}\right)$ and nitrogen $\left({ }^{14} \mathrm{~N} /{ }^{15} \mathrm{~N}\right.$ ), to determine the key reservoirs for these species (e.g., delivery as $\mathrm{N}_{2}$ or $\mathrm{NH}_{3}$ vastly alters the ${ }^{14} \mathrm{~N} /{ }^{15} \mathrm{~N}$ ratio in the giant planet's envelope). ${ }^{3} \mathrm{He} /{ }^{4} \mathrm{He}$ should be sampled with an accuracy of $\pm 3 \%$ (same as for the Galileo measurement). $\mathrm{D} / \mathrm{H},{ }^{12} \mathrm{C} /{ }^{13} \mathrm{C},{ }^{14} \mathrm{~N} /{ }^{15} \mathrm{~N}$ should be analyzed in the main host molecules with an accuracy of the order of $\pm 5 \%$;

- 2.2 Continuous measurements of atmospheric temperature and pressure throughout the descent to study (i) stability regimes as a function of depth though transition zones (e.g. radiative-convective boundary); (ii) accelerations; and (iii) the influence of wave perturbations, vertical winds and cloud formation on the vertical temperature profile. Continuous measurement of the conductivity profile to aid in understanding Saturnian lightning.

\section{Priority 3 measurements}

- 3.1 The isotopic ratios in $\mathrm{Ne}, \mathrm{Ar}, \mathrm{Kr}$ and $\mathrm{Xe}$ should be measured with accuracy better than $\pm 1 \%$, to give further constraints on the formation conditions of Saturn in the PSN. ${ }^{16} \mathrm{O} /{ }^{18} \mathrm{O}$ and ${ }^{17} \mathrm{O} /{ }^{18} \mathrm{O}$ with accuracy better than $\pm 1 \%$, should be sampled in order to investigate possible $\mathrm{O}$ isotopic variations throughout the solar system;

- 3.2 The vertical distributions of minor species to study vertical motions (e.g., $\mathrm{NH}_{3}, \mathrm{H}_{2} \mathrm{~S}$, $\mathrm{H}_{2} \mathrm{O}, \mathrm{PH}_{3}, \mathrm{AsH}_{3}, \mathrm{GeH}_{4}$ etc) should be measured from the tropopause to below the condensate clouds. $\mathrm{P} / \mathrm{H}, \mathrm{As} / \mathrm{H}$ and $\mathrm{Ge} / \mathrm{H}$ should be sampled with accuracy better than $\pm 10 \%$ (uncertainties close to solar abundances);

- 3.3 Measurements of the vertical structure and properties of Saturn's cloud and haze layers; including determinations of the particle optical properties, size distributions, number and mass densities, opacity, shapes and, potentially, their composition;

- 3.4 Determination of the vertical variation of horizontal winds during the descent. This includes a study of the depth of the zonal wind fields, and first measurements of middle atmospheric winds;

- 3.5 Thermal profile and heat budget in the atmosphere;

- 3.6 Measure accurate photometric light curve to probe for oscillations of the planet. 
Table 2 Suite of scientific instruments.

\begin{tabular}{|l|l|l|}
\hline Instrument & Location & Measurement \\
\hline Mass Spectrometer (MS) & Probe & $\begin{array}{l}\text { Elemental and chemical composition } \\
\text { Isotopic composition } \\
\text { High molecular mass organics } \\
\text { Pressure, temperature, density, molecular } \\
\text { weight profile, lightning } \\
\text { Measure winds, speed and direction } \\
\text { Chemical composition } \\
\text { Cloud structure, solid/liquid particles } \\
\text { Thermal/solar energy } \\
\text { Reasure winds and cloud structure } \\
\text { Detect oscillation signatures }\end{array}$ \\
$\begin{array}{l}\text { Nephelometer } \\
\text { Net-flux radiometer (NFR) } \\
\text { Camera }\end{array}$ & Probe & $\begin{array}{l}\text { Probe } \\
\text { Probe } \\
\text { Carrier }\end{array}$ \\
\hline
\end{tabular}


Table 3 Science Traceability Matrix.

\begin{tabular}{|c|c|c|c|c|c|}
\hline $\begin{array}{l}\text { Science } \\
\text { Goals }\end{array}$ & $\begin{array}{l}\text { Science } \\
\text { Objectives }\end{array}$ & \begin{tabular}{|l} 
Science \\
Prior- \\
ity
\end{tabular} & Science Questions & $\begin{array}{l}\text { Scientific Measure- } \\
\text { ments }\end{array}$ & Instrument \\
\hline $\begin{array}{l}\text { Understand } \\
\text { the } \\
\text { formation of } \\
\text { the Giant } \\
\text { Planets and } \\
\text { their roles } \\
\text { in the } \\
\text { evolution of } \\
\text { the solar } \\
\text { system }\end{array}$ & $\begin{array}{l}\text { Determine } \\
\text { the } \\
\text { composition } \\
\text { of Saturn's } \\
\text { well-mixed } \\
\text { atmosphere } \\
\text { beneath the } \\
\text { clouds }\end{array}$ & $2.1,3.1$ & $\begin{array}{l}\text { What is the abundance of } \\
\text { helium relative to } \mathrm{H}_{2} \text { ? } \\
\text { What are the well-mixed } \\
\text { abundances of the noble } \\
\text { gases? } \\
\text { What are the abundance } \\
\text { profiles of key cosmo- } \\
\text { genic species? } \\
\text { What are the most impor- } \\
\text { tant reservoirs for main } \\
\text { isotopes of H, He he- } \\
\text { lium, nitrogen, carbon, } \\
\text { oxygen, neon and heavy } \\
\text { noble gases? }\end{array}$ & $\begin{array}{l}\mathrm{He} / \mathrm{H}_{2} \text { ratio to an accu- } \\
\text { racy of } 2 \% \\
\mathrm{Ne} / \mathrm{H}, \mathrm{Ar} / \mathrm{H}, \mathrm{Kr} / \mathrm{H}, \mathrm{Xe} / \mathrm{H} \\
\text { to a precision of } \pm 10 \% \\
\mathrm{C} / \mathrm{H}, \mathrm{N} / \mathrm{H} \text { and } \mathrm{S} / \mathrm{H}: \pm 5 \% \\
\\
\begin{array}{l}{ }^{14} \mathrm{~N} /{ }^{15} \mathrm{~N},{ }^{12} \mathrm{C} /{ }^{13} \mathrm{C} \mathrm{D} / \mathrm{H}: \\
\pm 5 \%,{ }^{3} \mathrm{He} /{ }^{4} \mathrm{He}: \pm 3 \% \\
\mathrm{Ne}, \mathrm{Ar}, \mathrm{Kr} \text { and } \mathrm{Xe} \text { iso- } \\
\text { topes: } \pm 1 \%,{ }^{18} \mathrm{O} /{ }^{16} \mathrm{O} \\
{ }^{17} \mathrm{O} /{ }^{16} \mathrm{O}: \pm 1 \%\end{array}\end{array}$ & $\begin{array}{l}\text { MS, ASI, } \\
\text { RSE/AAbs } \\
\text { MS, ASI }\end{array}$ \\
\hline $\begin{array}{l}\text { Understand } \\
\text { Giant Planet } \\
\text { atmospheric } \\
\text { circulation, } \\
\text { the } \\
\text { processes } \\
\text { by which } \\
\text { energy is } \\
\text { transferred } \\
\text { outwards } \\
\text { from their } \\
\text { interior, and } \\
\text { the structure } \\
\text { of the cloud } \\
\text { layers }\end{array}$ & \begin{tabular}{|l|} 
Determine \\
the compo- \\
sitional, \\
thermal, and \\
dynamical \\
structure of \\
Saturn's \\
atmosphere
\end{tabular} & 3.4 & $\begin{array}{l}\text { What is the vertical } \\
\text { structure of Saturns at- } \\
\text { mospheric temperatures } \\
\text { and stability? } \\
\text { How do atmospheric } \\
\text { winds and wave phe- } \\
\text { nomena vary as a } \\
\text { function of depth? } \\
\text { How do convective mo- } \\
\text { tions and vertical mix- } \\
\text { ing shape the vertical } \\
\text { distribution of chemical } \\
\text { species? } \\
\text { What is the vertical } \\
\text { structure, composition } \\
\text { and properties of Sat- } \\
\text { urn's cloud and haze } \\
\text { layers? } \\
\text { What is the radiative en- } \\
\text { ergy balance of the atmo- } \\
\text { sphere? }\end{array}$ & $\begin{array}{l}1 \text { Pressure: } \pm 1 \% \text {, temper- } \\
\text { ature: } \pm 1 \mathrm{~K} \text { from the up- } \\
\text { per atmosphere to } 10 \text { bar } \\
\text { Profile of descent probe } \\
\text { telemetry Doppler fre- } \\
\text { quencies Zonal Winds: } \\
\pm 1 \mathrm{~m} / \mathrm{s} \text { from } 0.1-10 \text { bar } \\
\text { Vertical profiles of } \mathrm{NH}_{3}, \\
\mathrm{H}_{2} \mathrm{~S}, \mathrm{H}_{2} \mathrm{O}, \mathrm{PH}_{3}, \text { AsH } \\
\mathrm{GeH}_{4}, \mathrm{CO}: \pm 10 \% \\
1 \\
\\
\text { Particle optical proper- } \\
\text { ties, size distributions, } \\
\text { number and mass densi- } \\
\text { ties, opacity, shapes, and } \\
\text { composition } \\
\text { Up \& down visible } \\
\text { flux: } \sim 0.4-5 \mu \text { m; up \& } \\
\text { down IR flux: } 4-50 \mu \mathrm{m} ; \\
\lambda / \Delta \lambda \sim 0.1-100, \Delta \text { Flux } \\
\sim 0.5 \text { Wm }{ }^{-2} \\
\text { Long-term global pho- } \\
\text { tometry of the planet }\end{array}$ & $\begin{array}{l}\text { RSE/DWE } \\
\text { Camera } \\
\text { MS, ASI }\end{array}$ \\
\hline
\end{tabular}


Table 4 Summary of Parameters for Hera Science Instruments.

\begin{tabular}{|c|c|c|c|c|c|}
\hline & Mass & Size & $\begin{array}{l}\text { Power } \quad \mathrm{Re}- \\
\text { quirement }\end{array}$ & Data Rate & $\begin{array}{ll}\begin{array}{l}\text { Data } \\
\text { ume }\end{array} & \text { Vol- } \\
\end{array}$ \\
\hline Carrier Camera & $7.5 \mathrm{~kg}$ & $30 \times 14 \times 12 \mathrm{~cm}^{3}$ & $\sim 12 \mathrm{~W}$ & $100 \mathrm{Mb} / \mathrm{s}$ & $\begin{array}{l}6.8 \text { Mbytes } \\
\text { per image }\end{array}$ \\
\hline Probe Mass Spectrometer & $16 \mathrm{~kg}$ & $\begin{array}{l}24.5 \times 14.5 \times 22.9 \\
\mathrm{~cm}^{3}\end{array}$ & $\sim 68 \mathrm{~W}$ & $\sim 2 \mathrm{~kb} / \mathrm{s}$ & 10.7 Mbit \\
\hline Probe Atmospheric Structure Inst. & $1.5 \mathrm{~kg}$ & $20 \times 20 \times 20 \mathrm{~cm}^{3}$ & $\sim 10 \mathrm{~W}$ & $\sim 250 \mathrm{~b} / \mathrm{s}$ & 1.35 Mbit \\
\hline Radio Science & $1.5 \mathrm{~kg}$ & $\begin{array}{l}\sim 4 \mathrm{~cm} \text { diam } \times 14 \mathrm{~cm} \\
\text { length }\end{array}$ & $\begin{array}{l}\sim 3 \mathrm{~W} \\
\text { (warmup) }\end{array}$ & $\sim 2 \mathrm{~b} / \mathrm{s}$ & 10.8 kbit \\
\hline Net Flux Radiometer & $2.4 \mathrm{~kg}$ & $\begin{array}{l}11.3 \times 14.4 \times 27.9 \\
\mathrm{~cm}^{3}\end{array}$ & $\begin{array}{l}\sim 6.3 \mathrm{~W} \\
\text { (peak) } \\
\sim 4.7 \mathrm{~W} \\
\text { (basic) }\end{array}$ & $\sim 55 \mathrm{~b} / \mathrm{s}$ & $297 \mathrm{kbit}$ \\
\hline Nephelometer & $2.3 \mathrm{~kg}$ & & $\sim 3 \mathrm{~W}$ & $\sim 150 \mathrm{~b} / \mathrm{s}$ & 810 kbit \\
\hline
\end{tabular}


Table 5 Specifications of the MS elements.

\begin{tabular}{|c|c|c|}
\hline & Mass & Power \\
\hline TOF-MS & $\begin{array}{l}8.2 \mathrm{~kg} \text { (ion optical system, TOF electron- } \\
\text { ics, DPU, gas entrance system, pumping) }\end{array}$ & 25 watts (full operation) \\
\hline GSES & $\begin{array}{l}4.9 \mathrm{~kg} \text { (Cryotrap, heaters, valves, pump, } \\
\text { electronics) }\end{array}$ & $\begin{array}{l}20 \text { watts (maximum, nominally } 1 \mathrm{~W} \text { and } \\
\text { the ion pump power) }\end{array}$ \\
\hline RGS & $\begin{array}{l}1.2 \mathrm{~kg} \text { ( } 3 \text { reference gas containers, valves, } \\
\mathrm{NEG} \text {, ion pump and control electronics) }\end{array}$ & $\begin{array}{l}20 \text { watts (maximum before atmospheric } \\
\text { entry, nominally } 1 \mathrm{~W} \text { and the ion pump } \\
\text { power) }\end{array}$ \\
\hline TDS & $1.5 \mathrm{~kg}$ & 3 watts \\
\hline
\end{tabular}


Table 6 Specifications of the Nephelometer.

\begin{tabular}{|l|l|l|l|}
\hline & Mass & Power & Data Rate \\
\hline LOAC & $0.3 \mathrm{~kg}$ & 1 watt & $50 \mathrm{bps}$ \\
PAVO & $2 \mathrm{~kg}$ & 2 watt & $100 \mathrm{bps}(10$ optical heads $)$ \\
\hline
\end{tabular}


Table 7 List of filters.

\begin{tabular}{|c|c|c|}
\hline Name & Wavelength & Science \\
\hline MT2 & $727 \pm 10 \mathrm{~nm}$ & $\begin{array}{l}\text { Dynamics at the haze layers; cloud and } \\
\text { hazes vertical distribution }\end{array}$ \\
\hline CB2 & $760 \pm 10 \mathrm{~nm}$ & $\begin{array}{l}\text { Dynamics at the lower clouds; cloud and } \\
\text { hazes vertical distribution }\end{array}$ \\
\hline MT3 & $889 \pm 10 \mathrm{~nm}$ & $\begin{array}{l}\text { Dynamics at the haze layers; cloud and } \\
\text { hazes vertical distribution }\end{array}$ \\
\hline CB3 & $940 \pm 20 \mathrm{~nm}$ & $\begin{array}{l}\text { Dynamics at the lower clouds; cloud and } \\
\text { hazes vertical distribution }\end{array}$ \\
\hline UV/VIO & $380-420 \mathrm{~nm}$ & Cloud and hazes vertical distribution \\
\hline BLUE & $400-500 \mathrm{~nm}$ & $\begin{array}{l}\text { Outreach; cloud and hazes vertical distri- } \\
\text { bution; color }\end{array}$ \\
\hline RED & $650-750 \mathrm{~nm}$ & $\begin{array}{l}\text { Outreach; cloud and hazes vertical distri- } \\
\text { bution; color }\end{array}$ \\
\hline GREEN & $500-600 \mathrm{~nm}$ & $\begin{array}{l}\text { Outreach; cloud and hazes vertical distri- } \\
\text { bution; color }\end{array}$ \\
\hline
\end{tabular}


Table 8 Current TRL of the camera's elements.

\begin{tabular}{|c|c|c|c|}
\hline Sub-system L1 & TRL & Heritage & Remarks \\
\hline Camera & 3 & Corot, Rosetta/NAC & \\
\hline Filter wheel assembly & 3 & $\begin{array}{l}\text { Soho/coronograph, Lasco, } \\
\text { Herschel/Spire/FTS }\end{array}$ & $\begin{array}{l}\text { Long experience on } \\
\text { mechanisms }\end{array}$ \\
\hline Focal plane assembly & 3 & Corot, Euclid, Odin & \\
\hline Assembly detector & 5 & JUICE & $\begin{array}{l}\text { Fully qualified by time } \\
\text { needed for this program }\end{array}$ \\
\hline Electronic box & 3 & Herschel/Spire/FTS, Corot & \\
\hline
\end{tabular}


Table 9 Entry System Mass Estimates.

\begin{tabular}{|l|l|l|}
\hline $\begin{array}{l}\text { Entry Flight Path Angle } \\
\text { (EFPA), }\end{array}$ & -8 & -19 \\
\hline & \multicolumn{2}{|c|}{ Mass, kg } \\
Entry System (total mass) & 216 & 200 \\
Deceleration module & 92.5 & 76.5 \\
Forebody TPS (HEEET) & 40 & 24 \\
Afterbody TPS & 10.5 & 10.5 \\
Structure & 18.3 & 18.3 \\
Parachute & 8.2 & 8.2 \\
Separate Hardware & 6.9 & 6.9 \\
Harness & 4.3 & 4.3 \\
Thermal Control & 4.4 & 4.4 \\
Descent Module & 117.2 & 117.2 \\
Communication & 13 & 13 \\
C\&DH Subsystem & 18.4 & 18.4 \\
Power Subsystem & 19.8 & 19.8 \\
Structure & 30 & 30 \\
Harness & 9.1 & 9.1 \\
Thermal Control & 4.3 & 4.3 \\
Science Instrument & 28 & 28 \\
Separate Hardware & 0.9 & 0.9 \\
\hline
\end{tabular}

Note. Deceleration of module $1 \mathrm{~m}$ diameter aeroshell, $36 \mathrm{~km} / \mathrm{s}$ inertial velocity, $10^{\circ}$ latitude. The descent module mass estimate, except for the Science Instruments, are the same as that of Galileo Probe. Additional mass savings are likely when the descent system structure is adjusted for reduction in scale as well as entry $g$ load. Galileo design-to g-load was 350. Saturn probe entry g-load with 3-sigma excursions will be less than $150 \mathrm{~g}$ 's. 
Table 10 Entry g-loading, TPS mass comparison between HEEET and HCP, and recession mass loss for the limiting entry conditions.

\begin{tabular}{|l|l|l|l|l|l|l|l|l|l|l|}
\hline & $\begin{array}{l}\text { Inertial } \\
\text { velocity } \\
(\mathrm{Km} / \mathrm{s})\end{array}$ & $\begin{array}{l}\text { Geoc. } \\
\text { latitude }\end{array}$ & $\begin{array}{l}\text { Entry } \\
\mathrm{FPA}\left({ }^{\circ}\right)\end{array}$ & $\begin{array}{l}\text { Entry } \\
\text { Mass } \\
(\mathrm{kg})\end{array}$ & $\begin{array}{l}\text { Ballistic } \\
\text { Coeff. } \\
\left(\mathrm{kg} / \mathrm{m}^{2}\right)\end{array}$ & $\begin{array}{l}\text { Entry } \\
g \text {-load } \\
(g \text { 's })\end{array}$ & $\begin{array}{l}\text { HEEET } \\
\text { Mass } \\
(\mathrm{kg})\end{array}$ & $\begin{array}{l}\text { Carbon } \\
\text { Pheno- } \\
\text { lic Mass } \\
(\mathrm{kg})\end{array}$ & $\begin{array}{l}\text { Mass } \\
\text { loss } \\
\text { from } \\
\text { Reces- } \\
\text { sion(kg) }\end{array}$ & $\begin{array}{l}\text { TPS } \\
\text { Mass } \\
\text { loss/Entry } \\
\text { Mass }\end{array}$ \\
\hline 1 & 36.0 & 10.0 & -8.0 & 220 & 269 & 29 & 39.3 & 60.8 & 2.7 & $1.2 \%$ \\
2 & 36.0 & 10.0 & -19.0 & 220 & 269 & 131 & 23.8 & 33.9 & 2.6 & $1.2 \%$ \\
3 & 36.0 & 0.0 & -8.0 & 200 & 245 & 29 & 29.1 & 44.3 & 1.7 & $0.8 \%$ \\
4 & 36.0 & 0.0 & -19.0 & 200 & 245 & 127 & 18.7 & 27.1 & 1.6 & $0.8 \%$ \\
\hline
\end{tabular}


Table $11 \Delta \mathrm{V}$ and propellant budget.

\begin{tabular}{|c|c|c|c|c|c|c|}
\hline Manoeuvres & $\Delta \mathrm{V}$ & $\begin{array}{l}\text { Margin } \\
\text { on } \Delta \mathrm{V}\end{array}$ & Isp (s) & M final $(\mathrm{kg})$ & M initial (kg) & $\begin{array}{l}\text { Propellant } \\
\text { mass }(\mathrm{kg})\end{array}$ \\
\hline $\begin{array}{l}\text { Launcher dispersion } \\
\text { correction }\end{array}$ & 10 & $5 \%$ & 294 & 1271.9 & 1276.6 & 4.6 \\
\hline $\begin{array}{l}\text { VGAM and EGAM } \\
\text { nav allocation }\end{array}$ & 30 & $0 \%$ & 294 & 1258.8 & 1271.9 & 13.2 \\
\hline DSM before EGA & 190 & $5 \%$ & 294 & 1174.6 & 1258.8 & 84.1 \\
\hline $\begin{array}{l}\text { Retargeting at Saturn } \\
\text { before release }\end{array}$ & 15 & $100 \%$ & 294 & 1162.5 & 1174.6 & 12.2 \\
\hline $\begin{array}{ll}\text { Avoidance } & \text { after } \\
\text { probe release } & \end{array}$ & 80 & $5 \%$ & 294 & 896.0 & 922.6 & \\
\hline & & & & \multicolumn{2}{|c|}{$\begin{array}{c}\text { GNC }(9 \mathrm{~kg})+100 \% \text { margin }(\mathrm{kg}) \\
\text { Residuals mass }(\mathrm{kg}) \\
\text { Total propellant mass }(\mathrm{kg})\end{array}$} & $\begin{array}{l}18 \\
3 \\
161.7\end{array}$ \\
\hline
\end{tabular}


Table 12 Mass estimates.

\begin{tabular}{|l|l|}
\hline Hera mass budget & $(\mathrm{kg})$ \\
\hline Probe & 200 \\
Payload on carrier & 8 \\
Bus electronics & 48 \\
Communications & 55 \\
GNC sensors/actuators & 25 \\
Mechanisms for probe separation & 11 \\
Solar Array & 213 \\
Carrier batteries (primary+secondary) & 85 \\
Bi-propellant Propulsion S/S & 44 \\
Thermal Control & 31 \\
Harness & 55 \\
Structure & 154 \\
Nominal Dry Mass at Launch & 929 \\
System Margin 20\% & 186 \\
Total Dry Mass at Launch & $\mathbf{1 1 1 5}$ \\
MOM/MMH Propellant & 162 \\
Total Wet Mass at Launch & $\mathbf{1 2 7 7}$ \\
Total Launched (with adapter) & $\mathbf{1 3 8 7}$ \\
\hline
\end{tabular}


Table 13 Work Breakdown Structure for Hera Science Instruments.

\begin{tabular}{|c|c|c|c|}
\hline \multicolumn{2}{|c|}{ Instrument } & \multirow{2}{*}{\begin{tabular}{|l|} 
Lead \\
O. Mousis, PI (FR)
\end{tabular}} & \multirow{2}{*}{\begin{tabular}{|l} 
Support \\
L. Fletcher, Co-PI (UK); \\
R. Hueso (ES); F.-X. \\
Schmider (FR)
\end{tabular}} \\
\hline 1.0 & Cameras (on Carrier) & & \\
\hline 1.1 & $\begin{array}{l}\text { Camera optics \& me- } \\
\text { chanics }\end{array}$ & P. Levacher (FR) & \\
\hline 1.2 & $\begin{array}{l}\text { CMOS chip \& Electron- } \\
\text { ics }\end{array}$ & A. Holland (UK) & $\begin{array}{l}\text { J. Endicott (UK); M. } \\
\text { Leese (UK) }\end{array}$ \\
\hline 1.3 & Filter Wheels & R. Hueso (ES) & $\begin{array}{l}\text { C. Ortega (ES); M. A. } \\
\text { Carrera (ES) }\end{array}$ \\
\hline 1.4 & Electronics box & P. Levacher (FR) & \\
\hline 2.0 & $\begin{array}{l}\text { Probe Mass Spectrome- } \\
\text { ter (MS) }\end{array}$ & P. Wurz, PI (CH) & $\begin{array}{l}\text { J. H. Waite, Co-PI } \\
\text { (USA); }\end{array}$ \\
\hline 2.1 & $\begin{array}{l}\text { TOF-MS, MS Swiss ele- } \\
\text { ment }\end{array}$ & P. Wurz (CH) & \\
\hline 2.2 & GSES, MS US element & J. H. Waite (USA) & \\
\hline 2.3 & RGS, MS UK element & A. Morse (UK) & S. Sheridan (UK) \\
\hline 3.0 & \begin{tabular}{|lr} 
Probe & Atmospheric \\
Structure & Investigation \\
(ASI) & \\
\end{tabular} & F. Ferri, PI (IT) & $\begin{array}{l}\text { A. Colaprete, } \\
\text { (USA); } \quad \text { Go-PI } \\
\text { (AUT) }\end{array}$ \\
\hline 3.1 & Accelerometers (ACC) & & \\
\hline 3.2 & Pressure sensors (PPI) & & \\
\hline 3.3 & $\begin{array}{l}\text { Temperature } \quad \text { Sensors } \\
(\mathrm{TEM})\end{array}$ & & \\
\hline 3.4 & $\begin{array}{l}\text { Atmospheric Electricity } \\
\text { Package (AEP) }\end{array}$ & & \\
\hline 3.5 & ASI Processor (DPU) & & \\
\hline 4.0 & $\begin{array}{l}\text { Radio Science (Probe } \\
\text { and Carrier) }\end{array}$ & D. Atkinson, PI (USA) & T. Spilker (USA) \\
\hline 4.1 & $\begin{array}{l}\text { Doppler Wind Experi- } \\
\text { ment }\end{array}$ & D. Atkinson (USA) & M. Bird (DE) \\
\hline 4.2 & \begin{tabular}{|lr} 
Atmospheric & UHF, \\
Absorption/ $/ \mathrm{NH}_{3}$ & abun- \\
dance & \\
\end{tabular} & D. Atkinson (USA) & T. Spilker (USA) \\
\hline 5.0 & $\begin{array}{l}\text { Probe Net Flux Ra- } \\
\text { diometer (NFR) }\end{array}$ & M. Amato, PI (USA) & $\begin{array}{l}\text { S. Aslam (USA); C. } \\
\text { Nixon (USA) }\end{array}$ \\
\hline 5.1 & $\begin{array}{l}\text { Instrument: optics, elec- } \\
\text { tronics, mechanical }\end{array}$ & S. Aslam (USA) & M. Amato (USA) \\
\hline 5.2 & $\begin{array}{l}\text { Detector (Germany) and } \\
\operatorname{rad} \text { hard ROIC (USA) }\end{array}$ & & M. Amato (USA) \\
\hline 5.3 & Filters & S. Calcutt (UK) & \\
\hline 6.0 & Probe Nephelometer & Daphne Stam, PI (NL) & $\begin{array}{l}\text { J.-B. Renard, (FR); O. } \\
\text { Munoz (ES); D. Banfield } \\
\text { (USA) }\end{array}$ \\
\hline 6.1 & $\begin{array}{l}\text { Light Optical Aerosol } \\
\text { Counter (LOAC) }\end{array}$ & & \\
\hline 6.2 & PAVO Optics & C. Keller (NL) & F. Snik (NL) \\
\hline 6.3 & PAVO Detector \& Elect. & D. Stam (NL) & \\
\hline
\end{tabular}


Table 14 Costs for ESA (M€).

\begin{tabular}{|l|l|l|l|}
\hline $\begin{array}{l}\text { Soyuz Launch } \\
\text { Carrier Relay Spacecraft (CRSC) }\end{array}$ & & 75 \\
& $\begin{array}{l}\text { Management, Quality, Engineering } \\
\text { Assembly, Integration and Test }\end{array}$ & 50 & 174 \\
SVM sub-systems & 104 & \\
Hera Probe (descent module) & & 63 \\
Ground segment (MOC + SOC) & & 34 \\
Operations / exploitation & & 35 \\
Internal cost ESA & & 33 \\
Total ESA & & 414 \\
\hline
\end{tabular}


Table 15 Costs for NASA (M€).

\begin{tabular}{|l|l|}
\hline Solar arrays & 16 \\
Thermal Protection System & 10 \\
Aeroshell & 53 \\
Total NASA & 79 \\
\hline
\end{tabular}




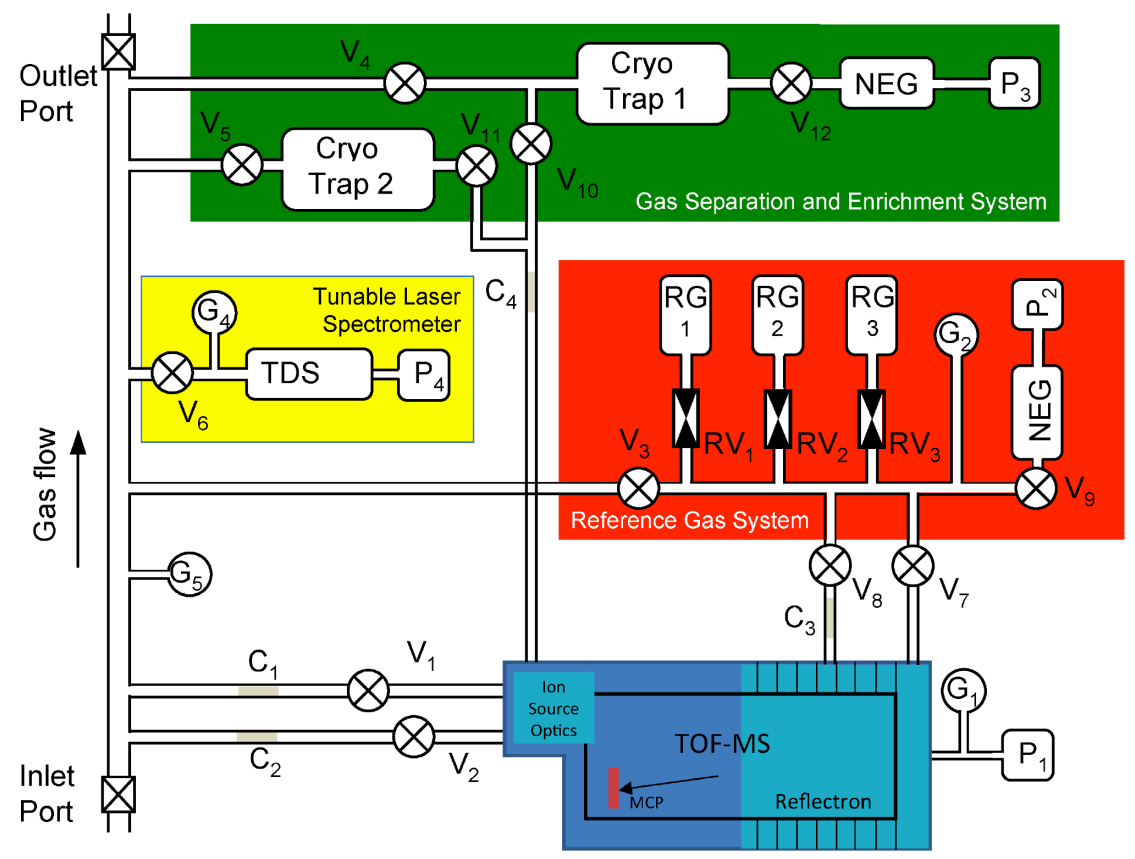

Fig. 1 Schematics of the Hera MS experiment, with the TOF-MS, the GSES, the RGS, and the TDS units. The elements are valves (V), regulating valves (RV), pressure gauges $(\mathrm{G})$, conductance limiters $(\mathrm{C})$, pumps $(\mathrm{P})$, gas reservoirs (RG), and non-evaporative getter (NEG). 


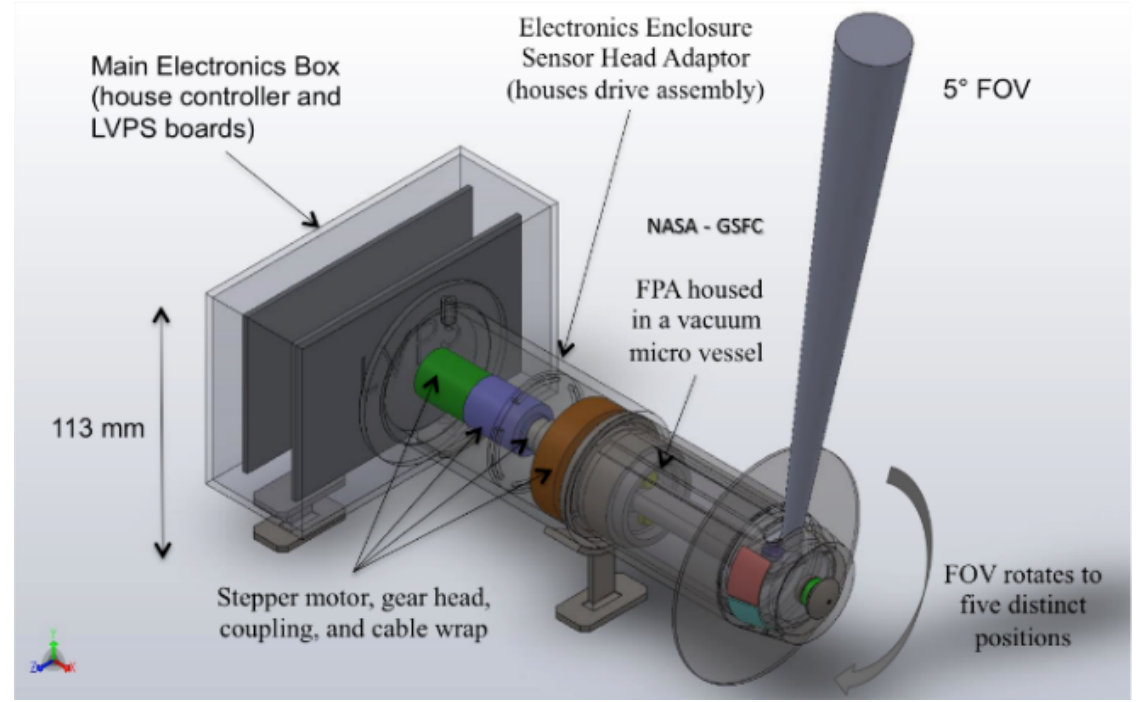

Fig. 2 NFR instrument concept showing a $5^{\circ}$ field-of-view that can be rotated by a stepper motor into five distinct look angles (NASA GSFC). 


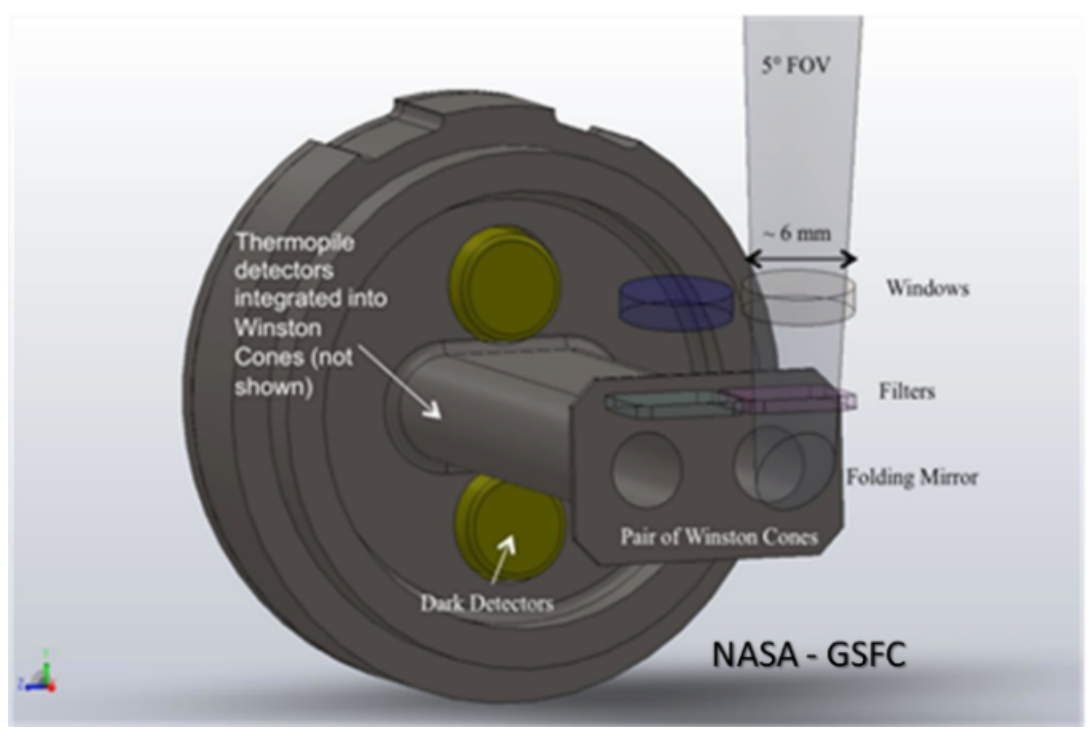

Fig. 3 NFR Focal Plane Assembly showing construction of Winston cones to limit FOV in each channel (NASA GSFC). 


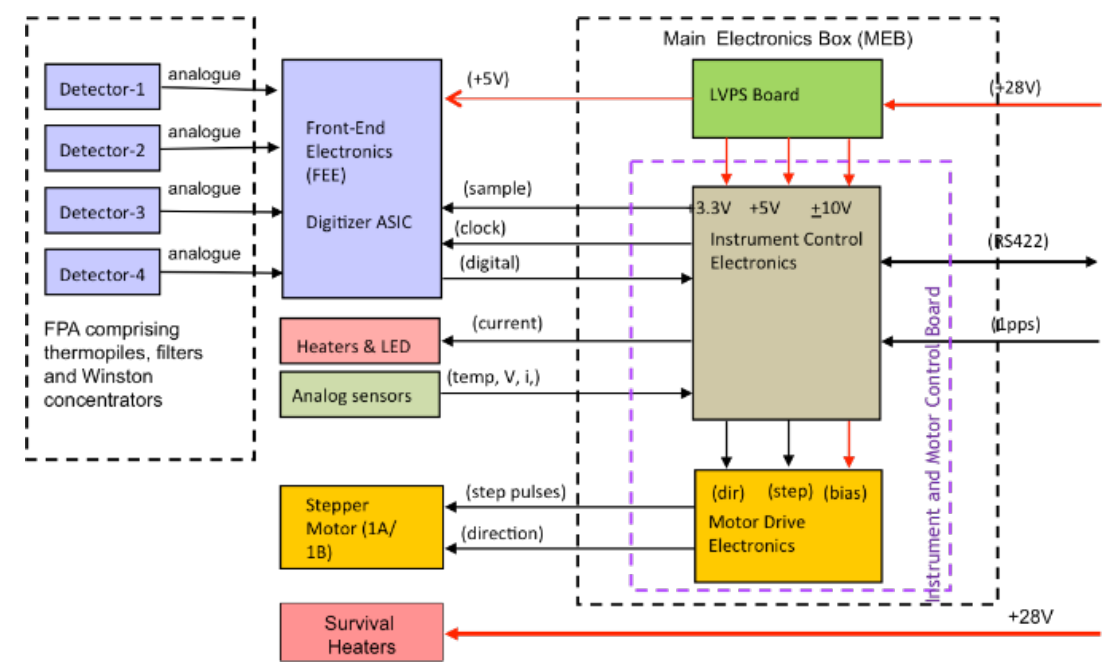

Fig. 4 Block diagram of the NFR showing the major subsystems and Probe interfaces. The redundant features are not shown (NASA GSFC). 


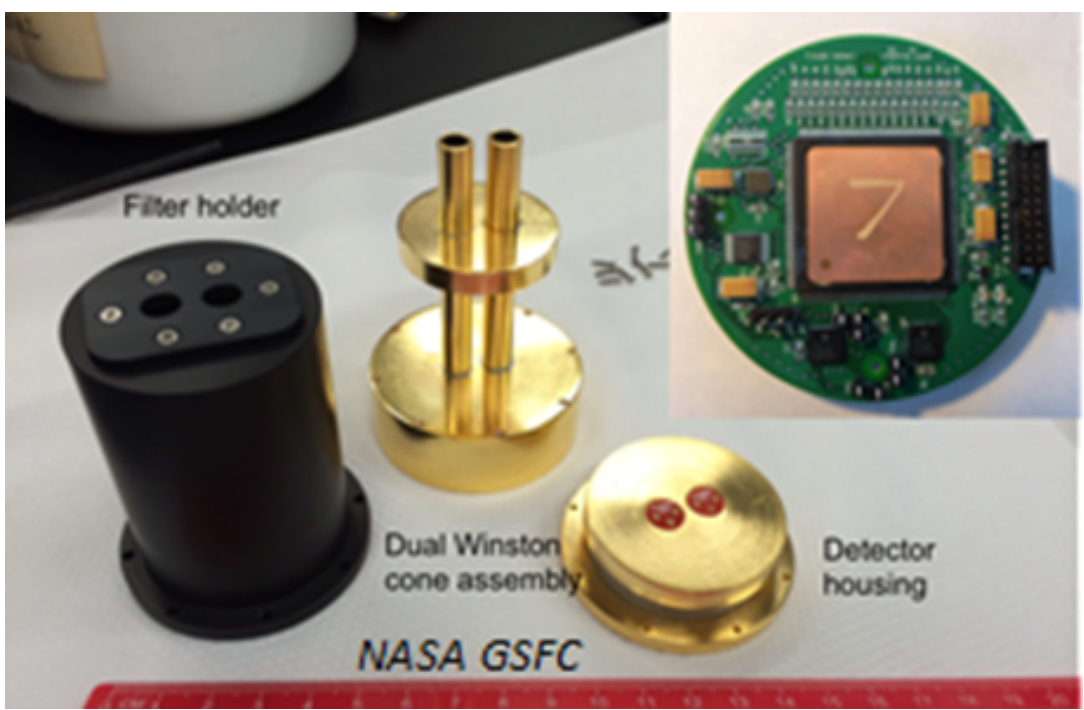

Fig. 5 NASA GSFC is testing early engineering models of the critical components the NFR. Dual Winston cone assembly and thermopile FEE readout (diameter $\sim 70 \mathrm{~mm}$ ) that uses a GSFC rad-hard mixed-signal ASIC (NASA GSFC). 


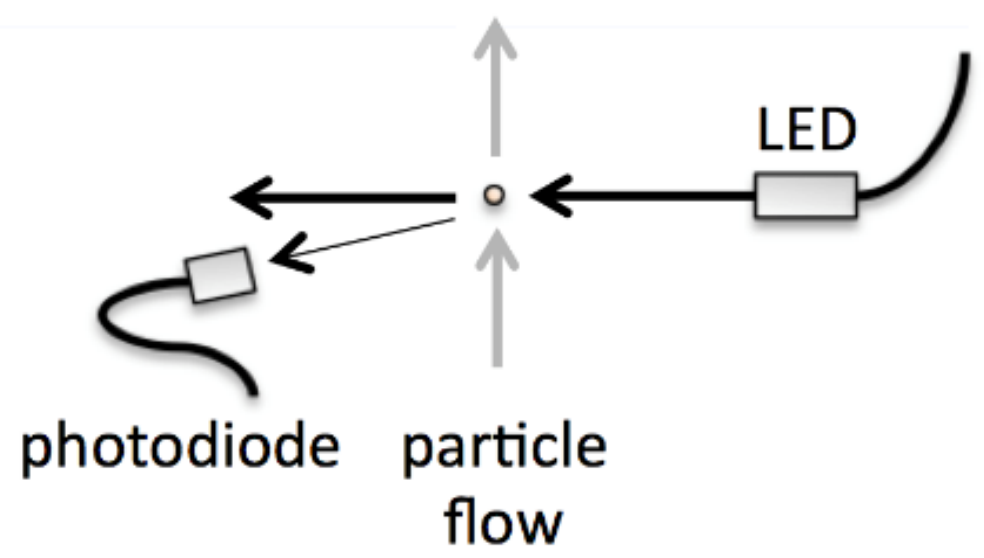

Fig. 6 Side-view of LOAC's design, with the particles crossing the LED light beam from below. The photodiode at a scattering angle $\Theta=12^{\circ}$ captures the forward scattered light. 


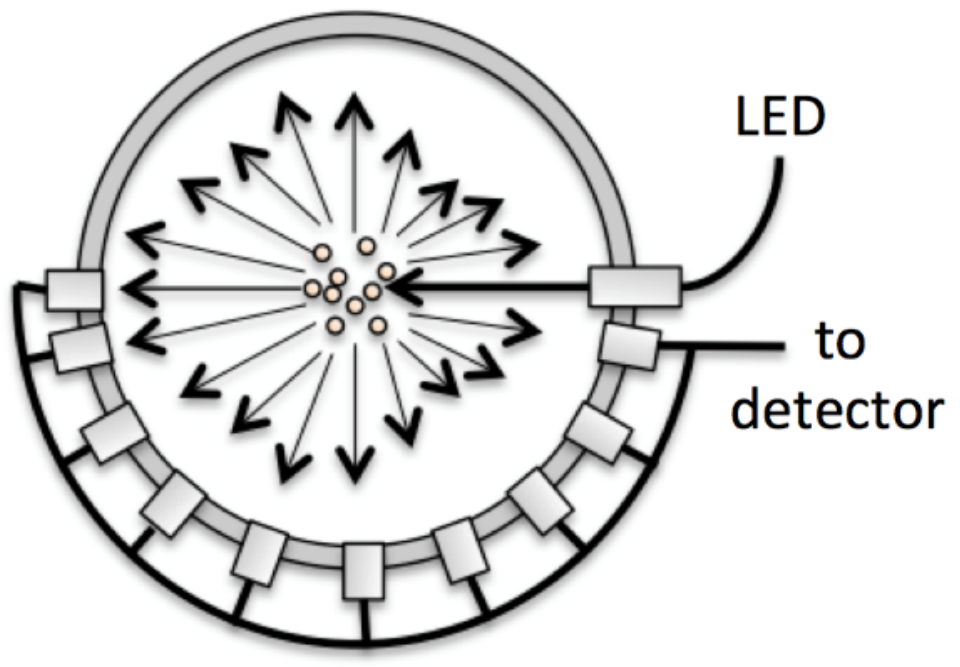

Fig. 7 Top-view of PAVO. One optical head captures non-scattered LED-light, and 9 heads capture scattered light. Fibres lead the modulated flux spectra from each head to the detector. 
The Hera Saturn Entry Probe Mission

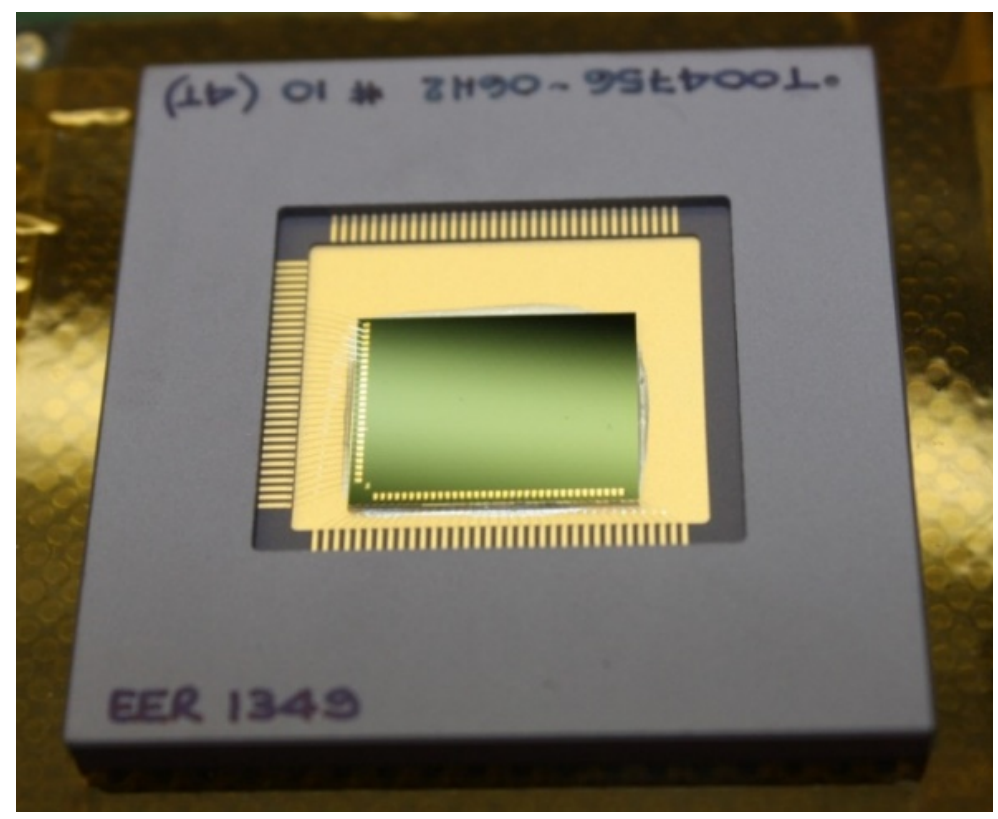

Fig. 8 CIS115 from e2v. 


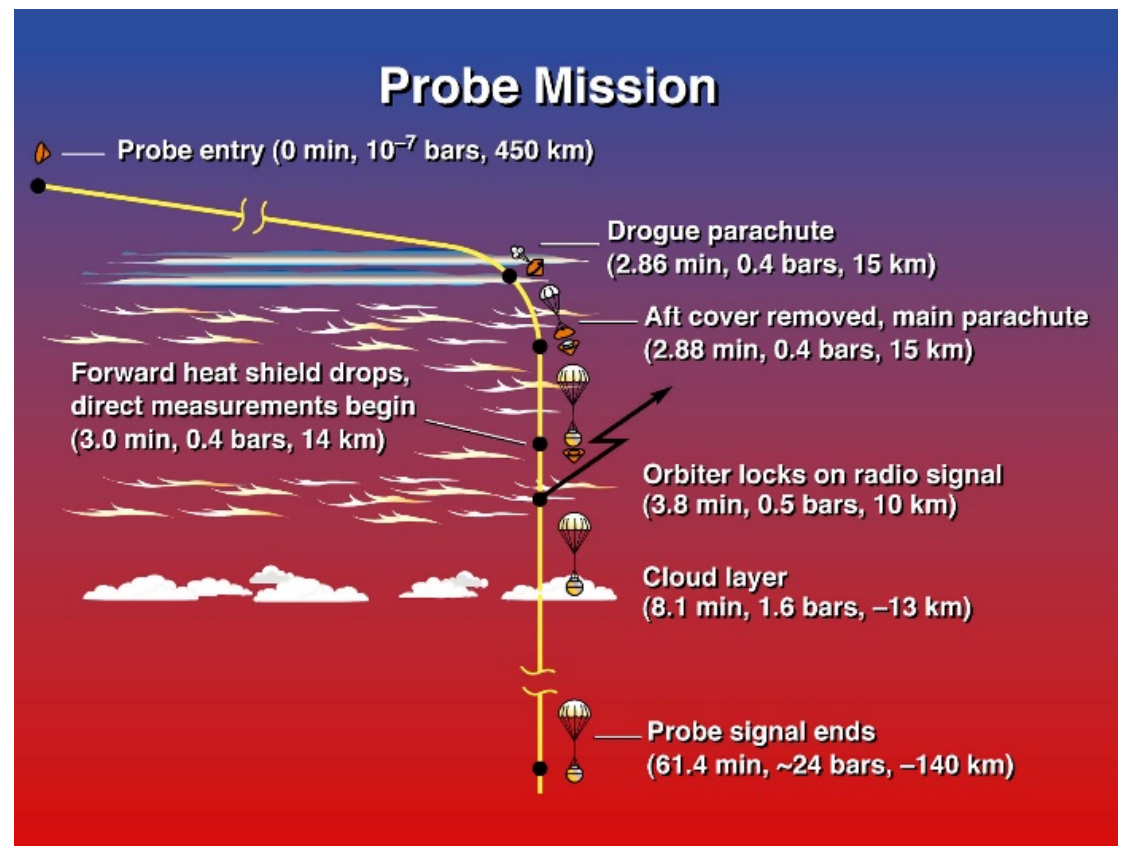

Fig. 9 Galileo entry, descent and deployment sequence shown above will be the basis for the proposed Saturn mission. 
SATURN MISSION DESIGN TRADES (EQUATORIAL +/- 10) LATTITUDE

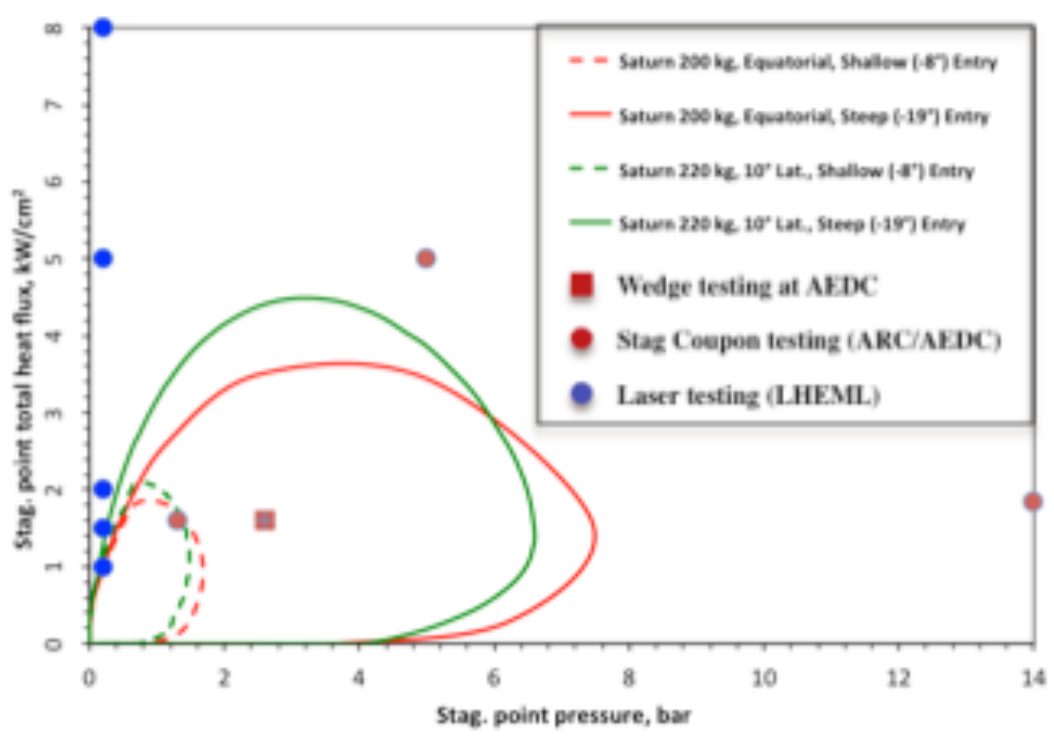

Fig. 10 Heat-flux and pressure (stagnation values) along four trajectories that bound the proposed Saturn mission is shown above along with arc-jet test conditions where HEEET has been tested. The HEEET acreage material shows exceptional performance with no failure even at extreme conditions (14 atmospheres and $2000 \mathrm{~W} / \mathrm{cm}^{2}$ ). 


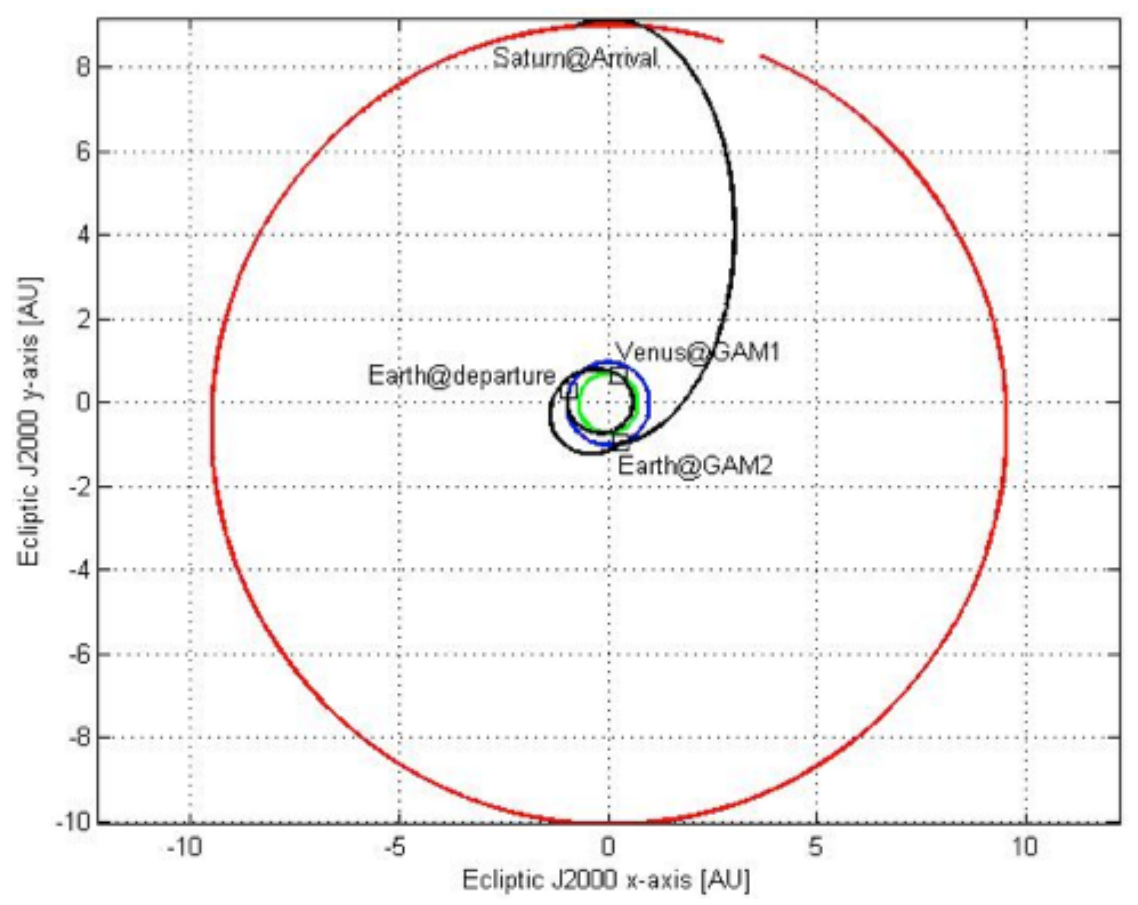

Fig. 11 Example of possible trajectory for Hera. 


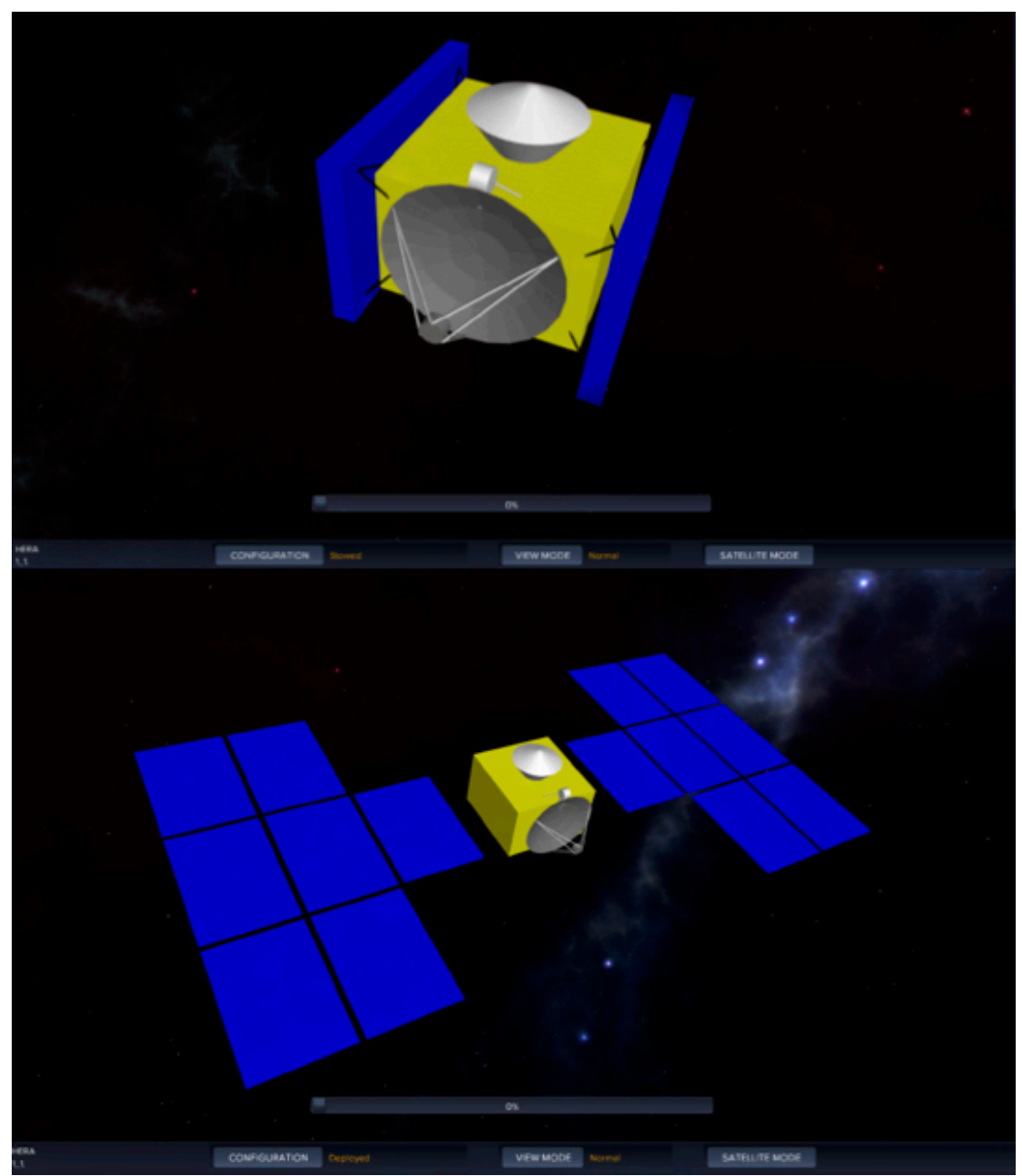

Fig. 12 7-panel solar array arrangement stowed (top) and deployed (bottom). 